ANL-80-87 MASTER 179

(1)

ANL-80-87

$$
R-2186
$$

POLYFAIL: A PROGRAM FOR IDENTIFICATION OF MULTIPLE FUEL FAILURES WITH GAS TAGGING

by

Kenny C. Gross

BASE TECHNOLOGY

BASE TECHNOLOGY

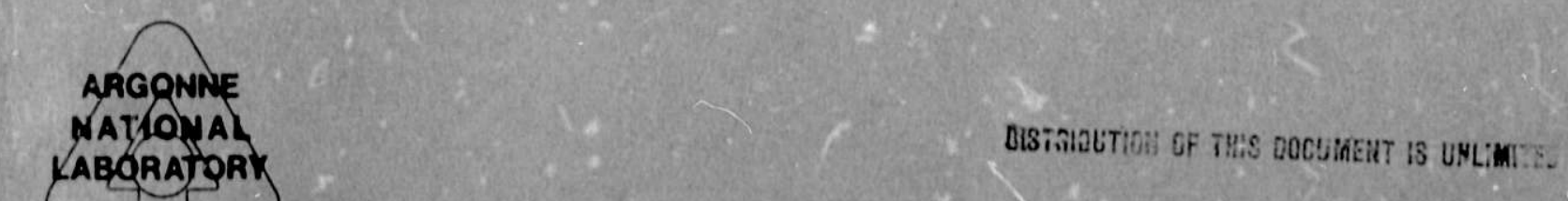

ARGONNE. NATIGNAL LABORATORY, ARGONNE, ILLINOIS

Prepared for the U. S. DEPARTMENT OF ENERGY

-undor Contract W-31-109-Eng-38 

TABLE OF CONTENTS

Page

ABSTRACT $\ldots \ldots \ldots \ldots \ldots \ldots \ldots \ldots \ldots \ldots \ldots \ldots \ldots \ldots \ldots \ldots \ldots \ldots \ldots \ldots \ldots \ldots$

I. INTRODUCTION. . . . . . . . . . . . .

I I. PROBlems associated With MUltiple tag Releases............. 8

A. Representation of Assembly Tag Nodes in Ratio Space........ 8

B. Geometry of Multiple Tag Releases................... 9

C. Calculation of Mixed-node Trajectories............... 12

D. Numerical Examples.......................... 14

III. IMPLEMENTATION OF THE BARYCENTRIC-COORDINATES TECHNIQUE....... 16

A. Triangular Simplex Method...................... 16

B. Systematic Procedurt for Resolution of Simultaneous

Failures.................................... 19

C. Exploiting the Concepts of Distance in Ratio Space.......... 23

D. Tetrahedral Simplex Method...................... 25

IV. IMPORTANCE OF ACCURACY IN SPECIFICATION OF ASSEMBLY TAG

COMPOSITIONS...................................... 27

v. TREATMENT OF BACKGROUND TAG ISOTOPES $\ldots \ldots \ldots \ldots \ldots \ldots \ldots \ldots \ldots \ldots . \ldots \ldots$

VI. $\quad$ SUSPECT-RANKIN : ALGORITHMS $\ldots \ldots \ldots \ldots \ldots \ldots \ldots \ldots \ldots \ldots \ldots \ldots$

A. Two-leaker Combinations......................... 29

B. Three-leaker Combinations...................... 30

C. Four-leaker Combinations...................... 31

vil, calculational strategres.......................... ?l

A. Limitations Imposed by Considerations of Linear

Independence................................... 31

B. Analytical Test for Coplanar Node Arrangements........... 32

C. Matrix-inversion Calculations..................... 33

D. Incorporation of Ratio-weighting Factors for Optimized

Sensitivity................................. 35

E. Preservation of Linearity...................... 36 
TABLE OF CONTENTS

$\underline{\text { Page }}$

VIII. SAMPLE PROBLEMS $\ldots \ldots \ldots \ldots \ldots \ldots \ldots \ldots \ldots \ldots \ldots \ldots \ldots \ldots \ldots \ldots \ldots \ldots$

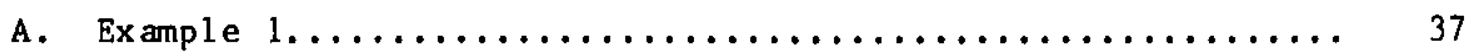

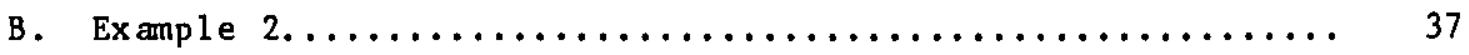

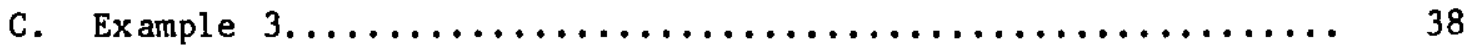

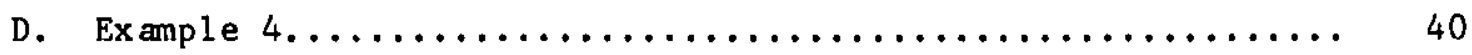

APPENDIXES

A. Proof of Geometric-invariance Property for Ratio-weighting Procedure................................. 42

B. Isotopic Ratios for Reference System of Tog Nodes........... 44

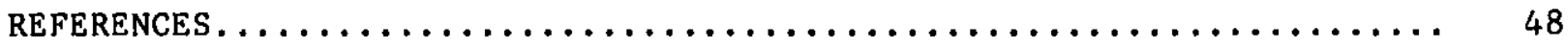




\section{LIST OF FIGURES}

1. Example System of Six Tag Nodes in $R_{1}-R_{2}$ Coordinate Piane...... 9

2. Example System of Six Tag Nodes, Showing Location of Second

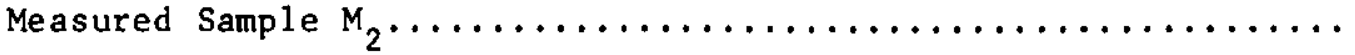

3. Definition of Distance Parameter $H_{i}$ for Determination of Most Likely Second Leaker.............................. 10

4. Positions of Measured Tag Nodes after Occurrence of a Third Tag

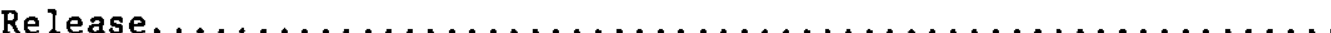

5. Trajectory of Mixed-tag Node for Simulated Three-leaker Example..

6. Three-node Triangle Used to Establish Transformation from Global (Cartesian) Coordinates to Barycentric Coordinates...............

7. Values Taken on by Components of $\overrightarrow{\mathrm{C}}_{3}$ in Varinus Regions of Simplex

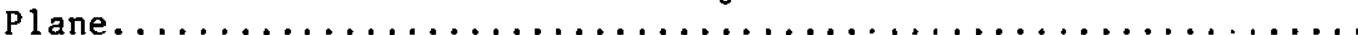

8. Flowchart for $\operatorname{Tr} i a n g u l a r$ Simplex Algorithm...............

9. Mixed-node Trajectory for Simulated Triple Failure of Assem-

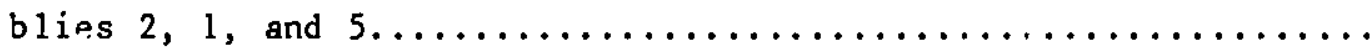

10. Relationship of Components of $\vec{C}_{3}$ Vector to Areas in Tag-node

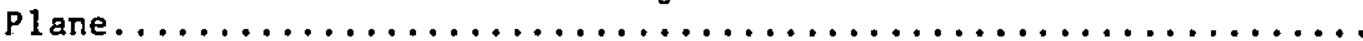

11. Relationship between $c_{i}$ and Triangle Altitudes when Measured

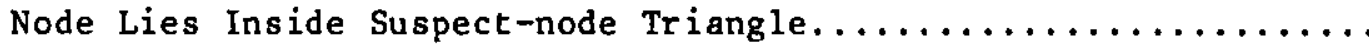

12. Relationship between $c_{i}$ and $T r i a n g l e$ Altitudes when Measured Node Lies Outside Suspect-node Triangle................... 24

13. Tetrahedral Node Arrangement in Three-dimensional Ratio Space.... 25

14. Displacement of Corrected Node Positions from True Node Positions as a Result of Composition Uncertainties..................

15. Alternative Ranking Algorithms for Resolution of Double Element

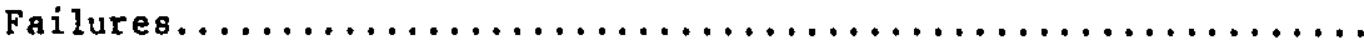

16. Relative Locations of Measured Nodes M1 and M2 un Simulated Mixed-node Trajectory Used in Example 3..................

B.1. Three-dimensional Plot of Reference Tag Nodes for ${ }^{78} \mathrm{Kr} /$

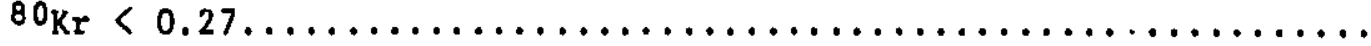

B.2. Three-dimensional Plot of Reference Tag Nodes for $0.27<{ }^{8} \mathrm{Kr} /$

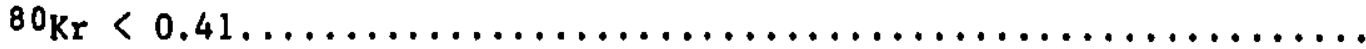

B.3. Three-dimensional Plot of Reference Tag Nodes for ${ }^{78} \mathrm{Kr} /$

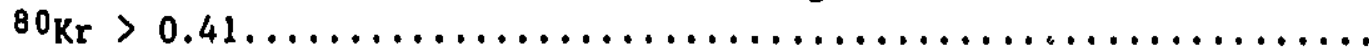




\section{LIST OF TABLES}

No.

Title

Page

I. Coordinates of Mixed-tag Node for Simulated Two-leaker Example... 14

II. Coordinates of Mixed-tag Node for Simulated Three-leaker

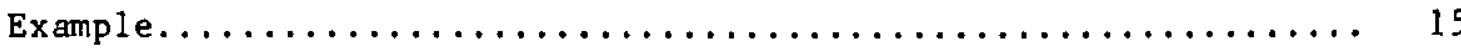

III. Geometric and Physical Properties of $\overrightarrow{\mathrm{C}}_{3}$ Solution Vector....... 18

IV. Barycentric-coordinates Technique Appl ied to Node $M_{1} \ldots \ldots \ldots 21$

V. Barycentric-coordinates Technique Applied to Node $M_{2} \ldots \ldots \ldots \ldots 22$

VI. Best Five One-leaker Combinations for Measured Node $M$ in

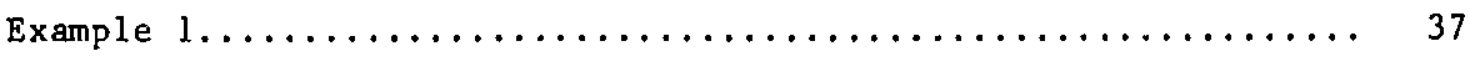

VII. Best Five One-leaker Suspects for Example $2 \ldots \ldots \ldots \ldots \ldots \ldots \ldots$

VIII. Best Ten Two-leaker Combinations for Example $2 \ldots \ldots \ldots \ldots \ldots$

IX. Best Five One-leaker Suspects for Measured Node Ml in Example 3.. 39

$X$. Best Ten Two-leaker Combinations for Measured Node Ml in

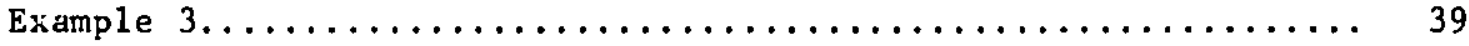

$X I$. Best Ten Three-leaker Cnmbinations for Measured Node M1 in

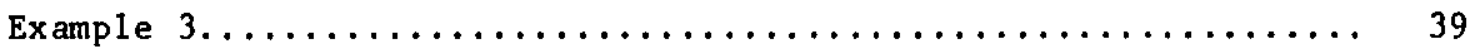

XII. Best Five One-leaker Stispects for Measured Node M2 in Example 3.. 39

XIII. Best Ten Two-leaker Suspects for Measured Node M2 in Example 3... 39

XIV. Best Ten Three-leaker Suspects for Measured Node M2 in Example $3 . \quad 39$

XV. Common Modes of Failure for Measured Tags M1 and M2 in Example 3.40

XVI. Compositions of Simulated Measured Tag Nodes Used in Example 4... 40

XVII. Summary of Computed Results from POLYFAIL for Example $4 \ldots \ldots \ldots, 41$

B.1. Tag Ratios for System of Tag Nodes Used in Example POLYFAIL

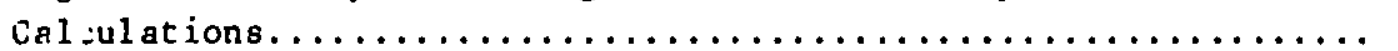




\title{
POLYFAII: A PROGRAM FOR IDENTIFICATION OF MULTIPLE FUEL FAILURES WITH GAS TAGGING
}

by

Kenny C. Gross

\begin{abstract}
This report describes the development of the computer code POLYFAIL for identification $0:$ fuel failures in fast reactors or light-water reactors that use gas tagging. POLYFAIL implements a sophisticated numerical algorithm known as the method of barycentric coordinates. The code can treat problems involving up to four simultaneous tag releases in a tagging system characterized by three independent tag ratios. The sensitivity of the multiple-failure-resolution technique has been optimized by incorporation of a newly developed ratio weighting scheme. Several example problems are provided to demonstrate operation of the code under singleleaker and various postulated multiple-leaker situations.
\end{abstract}

\section{INTRODUCTION}

One of the early difficulties encountered with the gas-tagging technique ${ }^{1-4}$ for identifying failed fuel assemblies* (assemblies containing one or more defective elements) involved the resolution of multiple, simultaneous failures. The term "simultaneous," as used here, refers to any failure that occurs while more than a negligible quantity of tag gas remains in the coolant system from one or more previous leakers.

Section II of this report recalls the problems associated with multiple tag releases by means of a small example and at the same time introduces the basic concepts of early vector techniques developed to treat these problems. Limitations in the vector-analysis approsch are described which severely restrict its userulness in treating simultaneous-release problems.

In Sec. III a new solution procedure that makes use of some powerful and efficient techniques from finite-element mathematics is presented and shown to overcome the limitations of the early approaches. The sew solution procedure

"The term "assembly" instead of "subassembly" will be used throughout this report to refer to a bundle of fuel elements. 
is known as the method of barycentric coordinates. 5 Specifically, the POLYFAIL code implements a three-dimensional realization of the barycentric-coordinates method, also known as the tetrahedral simplex technique. The code can treat problems involving up to four simultaneous tag releases in a tagging system characterized by three independent tag ratios.

Included in a final section of this report is a discussion of several example problems in which the general utility of the POLYFAIL code will become apparent.

\section{PROBLEMS ASSOCIATED WITH MULTIPLE TAG RELEASES}

In this section we review briefly the problems involved in attempting to identify the source of a new tag-gas release in the presence of one or more background tags. These background tags are underscood to include the natural background "tag," consisting of naturally occurring tag isotopos that are present as contaminants in the reactor cover gas; the tramp "tag," consisting of tag isotopes produced by the fissioning of tramp uranium; and assembly rags released from previous leakers and not yet purged from the system.

A. Representation of Assembly Tag Nodes in Ratio Space

Suppose that a tag release has occurred and the comosition of the released tag isotopes has been determined by mass spectromecry. What is now needed is a systematic procedure for making a quantitative comprison of the composition of the measured tag with the compositions of all assembly tags in the reactor to produce an objective ranking of the relative like!.ihood of the various possible modes of failure. The possible modes of failure that would be considered in the analysis would include single-leaker failures, wherein all the measured tag came from one defective assembly, and multiple-leaker failures, wherein vario's possible combinations of two or more assemblies might have released all or part of their tags simultaneously.

To make this problem analytically tractable, we begin by plotting the isotopic ratios of each tag in a Cartesian-coordinote system. The dimensionality of this Cartesian space is determined by the number of distinct ratios that can be formed from the constituent noble-gas isotopes. For example, a system of xenon tags created by blending together varying amounts of the three isotopes $124 \mathrm{Xe},{ }^{126} \mathrm{Xe}$, and $129 \mathrm{Xe}$ could be located conveniently in a twodimensional Cartesian plane by plotting $\mathrm{KL}=126 \mathrm{Xe} /{ }^{124} \mathrm{Xe}$ against $\mathrm{R} 2=129 \mathrm{Xe} /$ ${ }^{124} \mathrm{Xe}$. Each distinct assembly tag would map into a point, or node, in the ratio plane, thereby permitting us to exploit well-known principles from analytic geometry (or equivalently from linear algebra) to determine the most likely supect(s) in the event of a tag release. 
In Sec. II. B below, we introduce some intuitive ideas associated with the motion in ratio space of the node that represents a mixture of gases escaping from two or more elements simultaneously. In Sec. II.C, we put our intuitive ideas on a firm mathematical foundation and develop the ratio kinetics equations that will permit us to examine quantitatively the dynamic behavior of mixed-node trajectories in $\mathrm{N}$-dimensional ratio space.

\section{B. Geometry of Multiple Tag Releases}

We introduce here some geometric concepts needed to describe the position of the measured tag node relative to the configuration of one or more leaker nodes giving rise to that measured node. These concepts play a crucial role in the development of the ratio kinetics equations in a subsequent section.

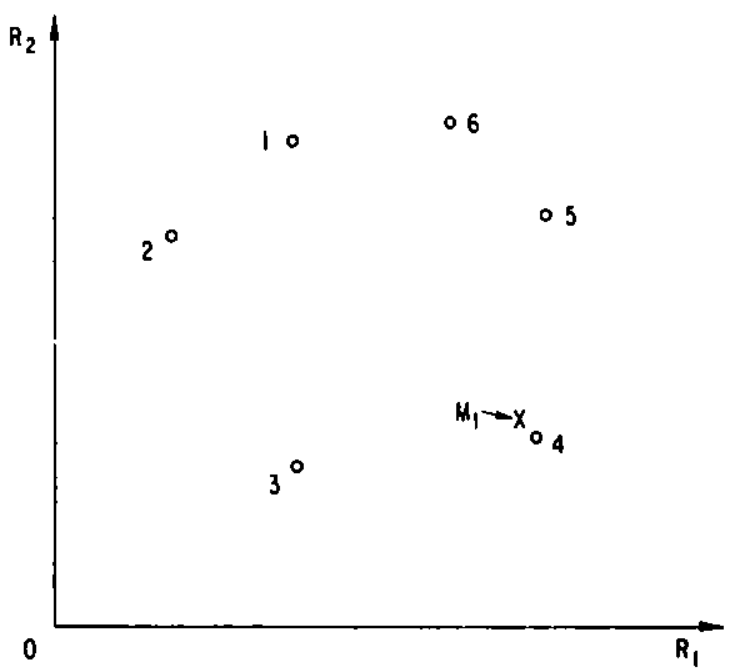

Fig. 1. Example System of Six Tag Nodes in $R_{1}-R_{2}$ (coordinate Plane
Illustrations will be helpful in visualizing the descriptions that follow. Thus, Fig. 1 illustrates an example system of six tag nodes whose characteristic tag ratios are plotted on a two-dimensional $\mathrm{Rl}-$ R2 ratio plane. Suppose that a tag release from one assembly has occurred and the location of the measured tag node as determined by mass-spectrometer analysis falls at point $M_{1}$ in the figure.

Ideally, the position of node $M_{\text {}}$ should coincide with the tag node associated with the leaking assembly. In a practical gas-tagging system, however, a number of effects may create small displacements in the positions of both the measured node and the assembly nodes. These effects

include the unavoidable experimental uncertainties associated with resolution of the mass-spectrometer detection equipment used to quantitate the isotopic ratios, uncertainties associated with the blending of the gases when the tags are first created, and, depending on the gases used, uncertainties in the noblegas isotopic ratios that arise from newtronics effects. ${ }^{6-8}$

If we are to specify a general procedure for identifying a single leaker, we must allow for the various node displacements just described. In a Cartesian ratio plane this can be accomplished conveniently by treating each assembly tag as a suspect and computing the vector distance from the measured node to all the suspect nodes. The suspect assembliec could then be ranked quantitatively in order of their increasing displacements from the measured node. For the elementary example shown in Fig. 1, this procedure would lead us to conclude that assembly 4 is the one most likely to be the source of measured tag $M_{1}$. 
If a second tag release were to occur while gas from the first release remained in the system, the position of the mixed-tag node (defined as the node representing the mixture of gases from the two leakers) would 1 ie on a 1 ine connecting the two tag nodes in the ratio plane. If the tag system comprises tag ratios $R_{1}$ and $R_{2}$ with the same isotope in the denominator of each ratio, then the mixed-tag node will always fall on a straight line connecting the two assembly nodes. If, on the other hand, the tag system comprises ratios characterized by more than one denominator isotope (e.g., $R_{1}=126 \mathrm{Xe} /{ }^{124} \mathrm{Xe}, \mathrm{R}_{2}=$ ${ }^{82} \mathrm{Kr} /{ }^{80} \mathrm{Kr}$ ), certain precautions must be taken, as outlined by McCormick in Ref. 2, to ensure that che position of the mixed-tag node falls on a straight line. It will be assumed throughout the present work that these precautions have been taken. (Otherwise, identification of double, triple, and higherorder failures would not be analytically tractable.)

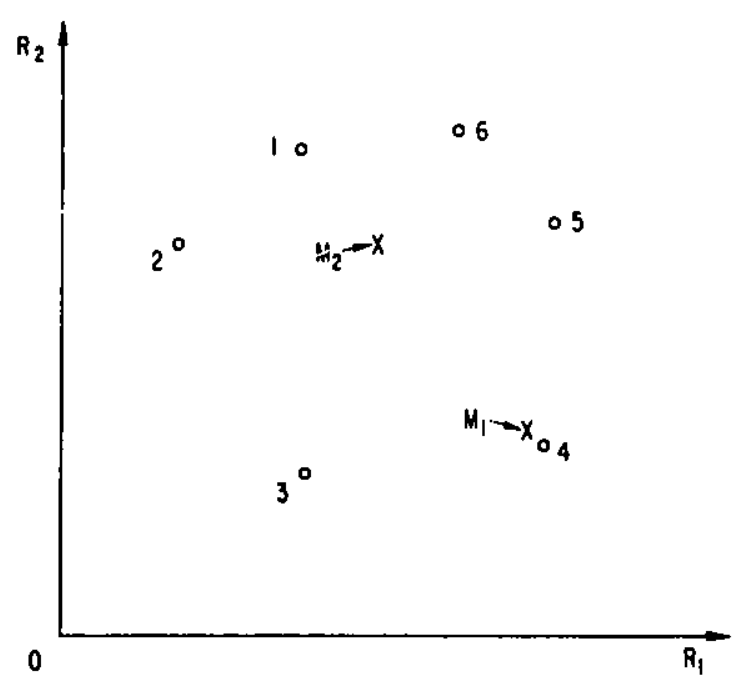

Fig. 2. Example System of Six Tag Nodes. Showing Location of Second Measured Sample $\mathrm{M}_{2}$
With these considerations in mind, let us assume that a second assembly in our example develops a leak and releases a portion of its tag while gas from assembly 4 (the first leaker) remains in the system. Position $\mathrm{M}_{2}$ in Fig. 2 depicts a possible location of the mixed-tag node for this case.

To identify the second leaker, we could take advantage once again of the geometry of the ratio plane and form $N-1$ vectors from node 4 (the first leaker) to each remaining suspect node, where $N$ is the total number of tags in the system. A vector is then drawn from node 4 to node $\mathrm{M}_{2}$, and elementary vector manipulations are used to compute the perpendicular distance, $H_{i}$, from node $M_{2}$ to each suspect vector (see Fig. 3). The suspect producing the smallest value of $H_{i}$ is the one considered most likely to contain the second leaker. For the system of nodes in Fig. 2, this procedure would indicate that assembly 1 contains the second leaker.

Fig. 3

Definition of Distance Parameter $\mathrm{H}_{1}$ for Determination of Most Llkely Second Leaker

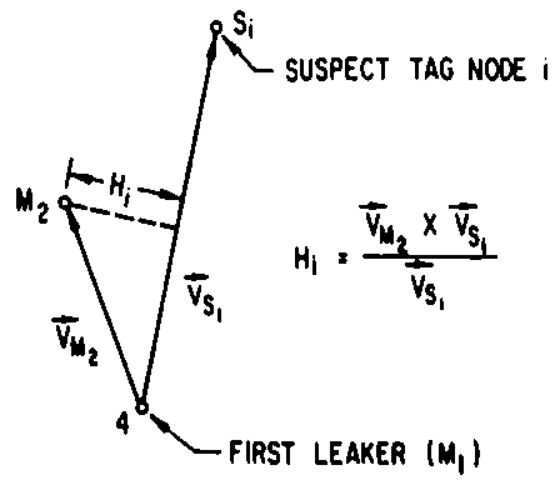


The procedure for identifying a third tag in the presence of two previously released tags has as its basis the experimentally verifiable principle that a node representing a mixture of three tags is confined to a triangle whose sides connect the three $t a g$ nodes. Thus, if a third gas sample, $M_{3}$, were analyzed and found not to fall on the line connecting nodes 4 and 1 , the analysis would be extended to search for a third leaker. In this case, N- 2 triangles would be formed using 1 ine segment $4-1$ as the base and the remaining suspects (i.e., nodes 2, 3, 5, and 6 in our example) as vertices (see Fig. 4). Vector techniques would again be used to determine which of the four sunpect triangles contains $M_{3}$. The triangles containing point $M_{3}$ would be considered feasible modes of failure, and parameters would be computed to determine the relative amounts of gas escaped from each leaker. Suspect triangles not containing $M_{3}$ would be eliminated from further consideration.

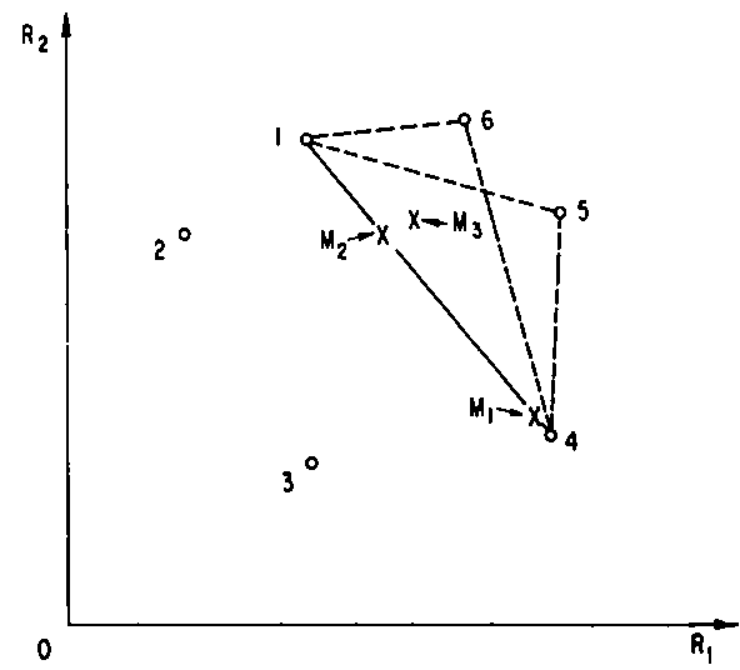

Fig. 4. Positions ol Measured Tag Nodes after Occurrence of a Third Tag Release
The situation depicted in Fig. 4 illustrates one of the limitations of a two-dimensional tag system: More than one triangle may contain the measured node (i.e., triangles 146 and 145 both contain $\mathrm{M}_{3}$ ). Under these circumstances and without additional information, there would be nothing more our $t a g$ analyses could tell us. Thus, for our example, we would know that assemblies 2 and 3 are not leaking, that assemblies 4 and 1 contain the first two leakers, and that there is a third defect which is equally likely to have occurred in assembly 5 or 6.

Early efforts to alleviate this problem of degenerate identification led

to three-ratio designs in which the system of tags was plotted in a threedimensional Cartesian space. In a three-dimensional system, the same vector techniques described above are used to identify a new leaker in the presence of two, one, or no previously identified leakers. The degenerate-identification problem is still possibility, but the likelihood of this situation arising is diminished because, for a given system of tags, the number of nodes that happen to be coplanar with the first two leakers and the point $M_{3}$ is reduced considerably. Locating the system of tags on curved surfaces ${ }^{2}, 9$ can further reduce the likelihood of this problem.

In three dimensions, one can, in principle, extend the identification techniques to locate a fourth leaker, once the previous three have been identified. In this case, use is made of the fact that the node representi 3 a mixture of four tags is confined to a tetrahedron whose vertices are the four tag nodes. 


\section{Calculation of Mixed-node Trajectories}

Up to this point the discussion has been strictly qualitative. Now that we have gained a physical understanding of the somewhat abstract concepts asso-" ciated with the "motion" in ratio space of the nole representing a mixture of tag gases, we will derive the equations required to compute the actual trajectories for that node.

Consider a three-ratio, two-gas, synergistic tag design such as that devised for the Fast Flux Test Facility. 10 We will label the two gases $A$ and $B$

and let the three coordinate ratios be identified with five isotopes: $x, y, z$, $a$, and $b$. Assume that $x, y$, and $z$ are isotopes of gas $A$, and $a$ and $b$ are isotopes of gas B. Suppose that a three-dimensional ratio space is defined in such a way that the three coordinate axes represent the respective ratios $x$ to $y, z$ to $y$, and a to $b$. These assumptions are necessary to render the theory as simple as possible while retaining considerable generality. Now, if $R 1, R 2$, and $R 3$ represent the three coordinates of a particular tag node, then at any time $t$ for any leaking element from the assembly corresponding to that node, the following equations must hold:

$$
\begin{aligned}
& x(t) / y(t)=R I, \\
& z(t) / y(t)=R 2,
\end{aligned}
$$

and

$$
a(t) / b(t)=R 3
$$

Furthermore, if we let $A(t)$ represent the toial quantity of gas $A$ remaining in the coolant system at time $t$, then we must have

$$
x(t)+y(t)+z(t)=A(t)
$$

A final constraint is imposed by the requirement that the volumes of the two gases used as the denominators in the three tag ratios be the same, i.e., that

$$
y=b,
$$

when the taga are charged to the fuel elements. This constraint is necessary to ensure that the tag ratios will lie along a straight line connecting the two tag nodes when two assemblies fail simultaneously. The constraint is satisfied in practice by varying the filling pressures of gases $A$ and $B$ when the two zases are initially blended. 2 
Equations 1-5 can be rearranged and expressed more ccncisely with matrix notation in the form

$$
\left(\begin{array}{ccccc}
1 & -R 1 & 0 & 0 & 0 \\
0 & -R 2 & 1 & 0 & 0 \\
0 & 0 & 0 & 1 & -R 3 \\
1 & 1 & 1 & 0 & 0 \\
0 & 1 & 0 & 0 & -1
\end{array}\right)\left(\begin{array}{c}
x(t) \\
y(t) \\
z(t) \\
a(t) \\
b(t)
\end{array}\right)=\left(\begin{array}{c}
0 \\
0 \\
0 \\
A(t) \\
0
\end{array}\right) .
$$

For demonstration purposes we will postulate that $A(t)$ is governed by an Arrhenius equation characterized by two rate coefficients $\lambda_{e}$ and $\lambda_{r}$, both of which have dimensions of inverse time. 11 The first of these , vefficients, $\lambda_{e}$, is the escape-rate coefficient for the tag gas from the fuel e, $\rightarrow$ to the coolant system. The second coefficient, $\lambda_{r}$, is the removal coe.i. it for noble gases of the species used to create the tags. In a press ric s.i Nater reactor, $\lambda_{r}$ is determined by the operating characteristics of the or - line gas stripper. 12 In a fast reactor, $\lambda_{r}$ is determined by the removal efficiency and turnover $t$ ime for the cover-gas cleanup system.

In accordance with the foregoing assumptions, $A(t)$ is computed from

$$
A(t)=\frac{\lambda_{e}}{\lambda_{r}-\lambda_{e}} A_{0}\left[\exp \left(-\lambda_{e} t\right)-\exp \left(-\lambda_{r} t\right)\right]
$$

For $\mathrm{N}$ simultaneously leaking elements, Eq. 6 is solved $\mathrm{N}$ times. The total volume of each individual isotope is then obtained by summation. Orice those volumes are known, the coordinates of the node representing the mixture of tag gas coming from the $\mathrm{N}$ elements can be computed with the equations

$$
\begin{aligned}
\alpha(t)= & \frac{\sum_{j=1}^{N} x_{j}\left(t-T_{j}\right)}{\sum_{j=1}^{N} y_{j}\left(t-T_{j}\right)}, \\
\beta(t)= & \frac{\sum_{j=1}^{N} z_{j}\left(t-T_{j}\right)}{\sum_{j=1}^{N} y_{j}\left(t-T_{j}\right)},
\end{aligned}
$$


and

$$
\gamma(t)=\frac{\sum_{j=1}^{N} a_{j}\left(t-T_{j}\right)}{\sum_{j=1}^{N} b_{j}\left(i-T_{j}\right)},
$$

where $T_{j}$ is the time of occurrence of the defect in element $j$ relative to the time of occurrence of the first defect.

The locus of points traced out by the three components functions $\alpha(t)$, $G(t)$, and $Y(t)$ completely determines the trajectory of the mixed-tag node.

\section{Numerical Examples}

A knowledge of the dynamics of tag-mixing phenomena is basic to an understanding of the techniques developed in later sections for deducing from a sequence of measured tags the identities of the leakers. Therefore, we pres ont here two numerical examples that amplify and illuminate the central ideas set forth in Sec. C above.

TABLE I. Coordinates of Mixed-tag Node for Simulated Two-leaker Example

\begin{tabular}{cccc}
\hline \multirow{2}{*}{$\begin{array}{c}\text { Time after } \\
\text { First } \begin{array}{c}\text { Defect, } \\
\text { min }\end{array}\end{array}$} & \multicolumn{3}{c}{$\begin{array}{c}\text { Computed Ratio } \\
\text { Coordinates }\end{array}$} \\
\cline { 2 - 4 } 5 & RI & R2 & R3 \\
\hline 10 & 1.00 & 1.00 & 1.00 \\
15 & 1.00 & 1.00 & 1.00 \\
20 & 1.05 & 1.05 & 1.05 \\
25 & 1.14 & 1.14 & 1.14 \\
30 & 1.19 & 1.19 & 1.19 \\
35 & 1.24 & 1.24 & 1.24 \\
40 & 1.28 & 1.28 & 1.28 \\
45 & 1.33 & 1.33 & 1.33 \\
50 & 1.36 & 1.36 & 1.36 \\
55 & 1.40 & 1.40 & 1.40 \\
60 & 1.44 & 1.44 & 1.44 \\
65 & 1.46 & 1.46 & 1.46 \\
70 & 1.52 & 1.52 & 1.52 \\
75 & 1.56 & 1.56 & 1.56 \\
80 & 1.60 & 1.60 & 1.60 \\
85 & 1.63 & 1.63 & 1.63 \\
90 & 1.67 & 1.67 & 1.67 \\
95 & 1.70 & 1.70 & 1.70 \\
100 & 1.74 & 1.74 & 1.74 \\
\hline & 1.76 & 1.76 & 1.76 \\
\hline & & &
\end{tabular}

The first example represents a double failure in which the coordinates of the nodes representing the two leaking assemlies are $(1,1,1)$ and $(2,2,2)$. The escape-rate coefficients for the two defective elements are specified arbitrarily to be $9.66 \times 10^{-4} \mathrm{~s}^{-1}$ and $3.0 \times 10^{-4} \mathrm{~s}^{-1}$. The onset of the defect in the second element is assumed to occur $13 \mathrm{~min}$ after the onset of the first defect. Table I lists the results of applications of Eqs. '5 and 7 . Examination of the results reveals that the mixed-node trajectory does indeed follow a straight 1 ine from the tag node for leaker 1 to the $t a g$ node for leaker 2 .

The second example deals with a twodimensional case of triple failure. The tag nodes corresponding to the thrse leaking assemblies have been chosen for sake of convenience to 1 ie in the $R 3=0 \mathrm{plane.}$ In the order of occurrence of their respective

assembly failures, the coordinates of the three tag nodes are $(1.0,1.0,0)$, $(3.0,1.0,0)$, and $(2.0,2.0,0)$. The escape-rate coefficient for the leak in assembly 1 is arbitrarily specified to be $5.0 \times 10^{-3} 8^{-1}$. Assembly 2 is assumed to develop a leak $8.0 \mathrm{~min}$ after the onset of the occurrence of the 
defect in assembly 1 . The escape-rate coefficitnt for this leak i. specified to be $8.33 \times 10^{-4} \mathrm{~s}^{-1}$. Finaily, assembly 3 is as sumed to develop a leak 25.0 min after the onset of the first defect, and the escape-rate coefficient for this leak is specified to be $3.25 \times 10^{-4} \mathrm{~s}^{-1}$.

Table II lists the computed results for this case. Figure 5 shows a plot of the computed trajectory. As ane would expect, the mixed-node trajectory begins at the $t a g$ node corresponding to leaker 1 and moves initially toward leaker 2. Then, after leaker 3 begins releasing its gas (at $t=25 \mathrm{~min}$ ), the trajectory leaves the line connecting 1 and 2 and moves out into the triangle toward leaker 3. As the gas in leaker 1 is gradually removed from the system, the trajectory is observed to merge with the line connecting nodes 2 and 3 . Finally, as the gas from the second leaker is removed from the system, the trajectory approaches its terminal point at node 3 .

TABLE II, Coordinates of Mixed-tag Node for Simulated Threeleaker Example

\begin{tabular}{ccc}
\hline $\begin{array}{c}\text { Time after } \\
\text { First Defect, } \\
\text { ni } n\end{array}$ & \multicolumn{2}{c}{$\begin{array}{c}\text { Computed Ratio } \\
\text { Coordinates }\end{array}$} \\
\cline { 2 - 3 } 10 & R1 & R2 \\
\hline 20 & 1.159 & 1.000 \\
30 & 1.668 & 1.000 \\
40 & 1.954 & 1.107 \\
50 & 2.143 & 1.262 \\
60 & 2.215 & 1.375 \\
70 & 2.242 & 1.473 \\
80 & 2.242 & 1.557 \\
90 & 2.227 & 1.630 \\
100 & 2.204 & 1.696 \\
110 & 2.177 & 1.753 \\
120 & 2.150 & 1.802 \\
130 & 2.123 & 1.843 \\
140 & 2.100 & 1.877 \\
150 & 2.080 & 1.905 \\
160 & 2.063 & 1.927 \\
170 & 2.049 & 1.944 \\
180 & 2.038 & 1.957 \\
\hline
\end{tabular}

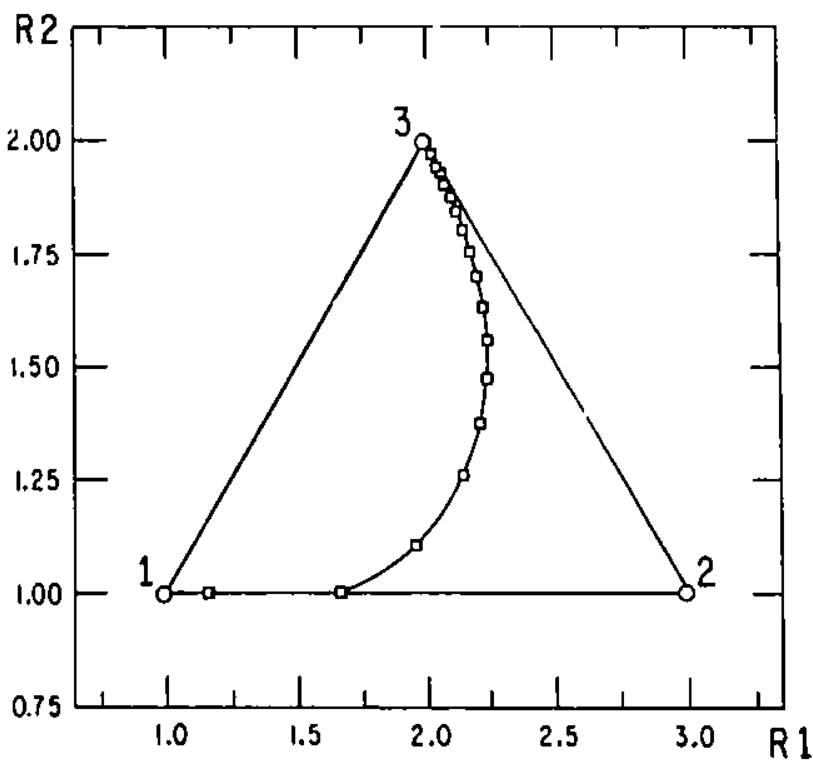

Fig. 5. Trajectory of Mixed-tag Node for Simulated Three-leaker Example

The situation described in the second heuristic example is, of course, an idealization insofar as it corresponjs to what we would "see" if we were able to measure the released tag isotopes instantaneously and with $100 \%$ accuracy. The actual situation as we are able to perceive it in practice may differ slightly for two reasons.

First, we do not know accurately the positions of the assembly tag nodes because of the effects of composition uncertainty mentioned previously. Second, even if we were able to obtain a measured sample and determine its composition with near-zero uncertainty, this sample would still not define a unique point on the node-trajectory curve-but rather it would represent an integral of some saction of that curve. This is because we cannot take instantaneous grab samples from the coolant system, but must accumulate the measured gas for some finite time. 


\section{IMPLEMENTATION OF THE BARYCENTRIC-COORDINATES TECHNIQUE}

Section II addressed the pioblems of determining the mixed-node trajectory in two- and three-dimensional ratio space, given the number of failed elements and the coordinates of their respective tag nodes. With that discussion as motivation, attention is now focused on the reverse problem, that which the POLYFAIL code was developed to solve. Specifically, the goal is to deduce from two or more measured nodes on the mixed-node trajectory the unambiguous identities of the active leakers.

The analytical technique developed to treat this problem is called the method of barycentric coordinates. Sections III.A and III.D below summarize the two- and three-dimensional realization of the barycentric-coordinates technique, also known, respectively, as the triangular and tetrahedral simplex methods. ${ }^{4}$ Readers interested in the formal development of the general Ndimensional barycentric-coordinates techrique are referred to Ref. 5 .

\section{A. Triangular Simplex Method}

The triangular simplex method is most clearly introduced through an elementary example. Thus, consider the three tag nodes plotted in Fig. 6. These three tag nodes define a node triangle in which we desire to define a

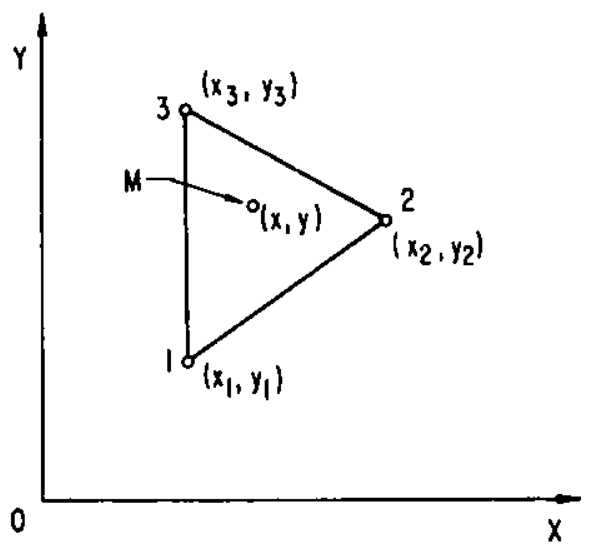

Fig. 6. Three-node Triangle Used to Establish Transformation from Global (Cartesian) Coordinates to Barycentric Coordinates barycentric-coordinates system.13-15 The goal is to select three weighting functions $c_{1}, c_{2}$, and $c_{3}$ to describe the coordinates of any point $M$ that falls inside, outside, or on the edge of the triangle.

We will let the original Cartesian coordinates of $M$ be $(x, y)$ and require that these coordinates be linearly related to the new coordinates by the equations

$$
x=c_{1} x_{1}+c_{2} x_{2}+c_{3} x_{3}
$$

and

$$
y=c_{1} y_{1}+c_{2} y_{2}+c_{3} y_{3} \text {. }
$$

The coordinates $c_{1}, c_{2}$, and $c_{3}$ may be interpreted as weighting functions relating the coordinates of the three tag nodes to the coordinates of any point in the plane. The desirable property of each weighting function is that the value of $c_{i}$ should be unity at node $i$ and zcro at the other two nodes. This condition is imposed by requiring that the weighting functions sum to unity, that is,

$$
c_{1}+c_{2}+c_{3}=1 \text {. }
$$


From Eq. 13 it is clear that only two of the barycentric coordinates can be independent, just as in the original ratio system, where there are only two independent coordinates.

Using matrix notation, we may cast Eqs. 11 and 12 in the form

$$
\left(\begin{array}{ccc}
x_{1} & x_{2} & x_{3} \\
y_{1} & y_{2} & y_{3} \\
1 & 1 & 1
\end{array}\right) \vec{c}_{3}=\left(\begin{array}{l}
x \\
y \\
1
\end{array}\right)
$$

where

$$
t_{3}=\left(\begin{array}{l}
c_{1} \\
c_{2} \\
c_{3}
\end{array}\right)
$$

Equation 14 exhibits at once both the power and the :implicity of the barycentric-coordinates technique. Because of the unique properties of the $t_{3}$ vector, one can perform the trivial inversion of Eq. 14 to determine $\vec{C}_{3}$ ard obtain all the infcrmation that can be extracted from a given mass-spectrometer

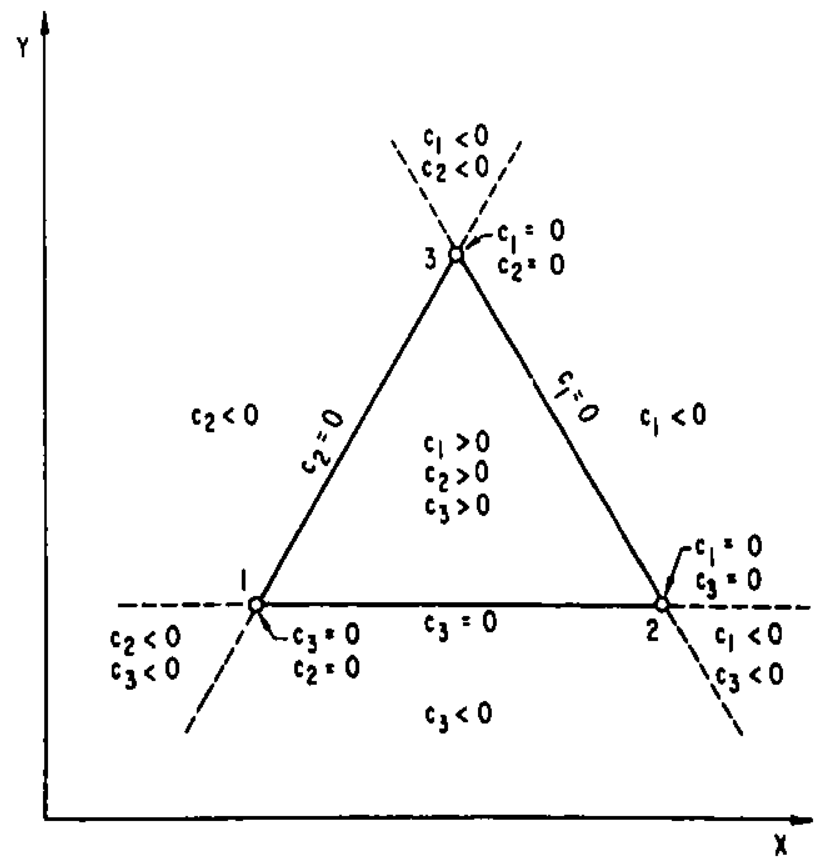

Fig. 7. Values Taken on by Components of $\overrightarrow{\mathrm{C}}_{3}$ in Various Reglons of Simplex Plane reading regarding which of the three suspect assemblies is leaking and which are not. Moreover, if more than one suspect is leaking, the $\vec{C}_{3}$ vector will also provide the relative amounts of tag gas (and hence also fission gas) that have escaped from each leaking assembly.

The properties of the components of $\mathrm{C}_{3}$ and the relation between the global coordinate system (i.e., the Cartegian ratio plane) and the local, barycentric-coordinates system are illustrated graphically in Fig. 7 for a general triangular simplex.

As described in Sec. II, the problem of determining which of three suspect assemblies is leaking and which are not is equivalent to determining where the measured tag node

falls with respect to the triangle whose vertices are the three ouspect nodes. This information can be obtained from the properties displayed in Fig. 7 by simply testing the values of the three components of $t_{3}$. 
For example, in the simplest case, in which only one of the three suspect assemblies is leaking, the component of $\vec{C}_{3}$ corresponding to that assembly will be identically 1 , while the remaining two components will be zero. Thus, if we determined that, say, $c_{2}$ equals 1 with $c_{1}$ and $c_{3}$ both zero, we would know that assembly 2 had a defective fuel element and that it was the only assembly containing a leaker. Geometr $711 y$, this would mean that the measured tag node coincides with node 2 in $\}$ ?. 7 .

Now suppose that one of the $c^{\prime} s$ is computed to be zero while the other two $c^{\prime} s$ have values between 0 and 1 . In this case, the assemblies corresponding to the two nonzer. components are both leaking tag gas while the assembly corresponding to the zero component is not. Thus, if it were found that, say, $c_{3}=$ 0 , while $0<c_{1}<1$ and $0<c_{2}<1$, we would know that assemblies 1 and 2 both contained leakers and assembly 3 could be eliminated as a suspoct. Geometrically, this situation would arise if the measured tag node were to fall on the line connecting nodes 1 and 2 in Fig. 7 .

If all three assemblies are leaking simultaneously, then all three $c$ 's in Eq. 15 will have values that are nonzero and nonnegative (i.e., by Eq. 13, this is equivalent to saying that all three c's will have values between 0 and 1 ). In this case it also means that the measured tag node falls inside the triangle whose vertices are the three tag nodes.

Finally, if any componest of $\overrightarrow{\mathrm{C}}_{3}$ is found to have s negative value, then at least one other assenbly (other than, ur possibly in addition to, the three assemblies currently beirg considered as suspect) is leaking. This case occurs when the measured tag node falls outside the triangle whose sides connect the three tag nodes.

Table III summarizes the various properties of the components of the $\overrightarrow{\mathrm{C}}_{3}$ vector that enable one to determine which of three given suspect assemblies is leaking and which are not. The table shows the physical interpretations and the corfesponding geometric interpretations for the various possible outcomes of the $\mathrm{C}_{3}$ calculation.

TABLE III. Geometric and Phyaical Properties of $\vec{C}_{3}$ Solution Vector

\begin{tabular}{|c|c|c|}
\hline $\begin{array}{l}\text { Outcome of } \vec{c}_{3} \\
\text { Determinat ion }\end{array}$ & Physical Interpretation & Geometric Interpretation \\
\hline $\begin{array}{l}c_{i}=1 \\
\because j=0 \\
i k=0\end{array}$ & Assembly i leaking & $\begin{array}{l}\text { Meagured node coincides with } \\
\text { tap node } i\end{array}$ \\
\hline $\begin{array}{l}\quad c_{i}-0 \\
\text { and } 0<c_{j}<1 \\
\text { and } 0<c_{k}<1\end{array}$ & $\begin{array}{l}\text { Asaemblies } j \text { and } k \\
\text { leaking inultaneously }\end{array}$ & $\begin{array}{l}\text { Measured node fallo on line } \\
\text { connecting nodes } j \text { and } k\end{array}$ \\
\hline $\begin{array}{l}0<c_{i}<1 \\
\text { and } 0<c_{j}<1 \\
\text { and } 0<c_{k}<1\end{array}$ & $\begin{array}{l}\text { Assembliea } i, j, \text { and } k \\
\text { leaking oimultaneously }\end{array}$ & $\begin{array}{l}\text { Measured node confined to } \\
\text { triangle whose sides connect } \\
\text { nodes } i, j \text {, and } k\end{array}$ \\
\hline $\begin{array}{l}c_{i}<0 \\
\frac{\text { or }}{\text { or }} c_{j}<0 \\
c_{k}<0\end{array}$ & $\begin{array}{l}\text { Aesemblies } i \text { and/or } j \text { and/or } k \\
\text { could be leakink, but at least } \\
\text { one other assembly must be } \\
\text { leaking ollewhere in syetem }\end{array}$ & $\begin{array}{l}\text { Measured node lies outaide } \\
\text { triangle whose aides connect } \\
\text { nodes } i, j \text {, and } k\end{array}$ \\
\hline
\end{tabular}


B. Systematic Procedure for Resolution of Simultaneous Failures

The stated properties of the componints of the $\vec{C}_{3}$ vector have been incorporated into a systematic algorithm for iinding wich assemblies in a group of tagged assemblies are leaking and which are not. Figure 8 depicts a simplified flowchart for the algorithm. As the figure shows, the algorithm consists of successively applying the tests from Table III to every possible combination of assemblies taken three at a time. We illustrate the procedure with an examnle that considers a group of five assemblies whose tag nodes aie in a plane as shown in Fig. 9. This example is trivial in theory, but it reveals the essential features of the general three-dimensional techniques to be discussed later.

The coordinates of the five $t a g$ nodes for this example are as indicated in the figure. To simulate a multiple failure, the coordinates of nodes 1,2 , and 5 were input into Eqs. 6-10 along with some arbitrarily chosen leak-rate coefficients. The resulting mixed-node trajestory is illustrated with the dotted curve connecting nodes 2 and 5 (cf. Fig. 5). This curve depicts a possible mixed-node trajectory that could result from simultaneous releases from assemblies 2, 1 , and 5 . Straight lines connecting the five tag nodes are shown to aid in visualizing the boundaries of the various suspect triangles considered below.

Two points have been indicated by $x^{\prime} s$ on the node-trajectory curve. These points, labeled $M_{1}$ and $i_{2}$, liave been selected to represent two measured $t a g$ nodes; that is to say that ve are assumirig that two gas samples have been separated from the coolant system and that, when plotted cn the X-Y ratio plane, the isotopic compositions for the first sample are those ior $M_{1}$, and the isotopic compositions for the second sample are those for point $M_{2}$, in this simulated mode of failure.

Before proceeding further, we should point out that, for this simple example, we could probably just as well have selected $M_{1}$ and $M_{2}$ arbitrarily instead of using the equations from Sec. II to simulate a failure and compute a trajectory. However, in a larger, three-dimensional system of tags, such an arbitrary selection of measured tag nodes might not correspond to any physically real mode of failure, and the multiple-failure analysis technique cnuld yield degenerate results.

Beginning with $M_{1}$, the properties in Table III are epplied to determine which combination of three or less assemblies could have failed to yield $M_{1}$. Table IV shows the results of this simplified analysis. The first column lists each suspect triangle. The vertices of these triangles are obtained by simply writing down all distinct combinations of the five tag nodes taken three at $a$ time. 


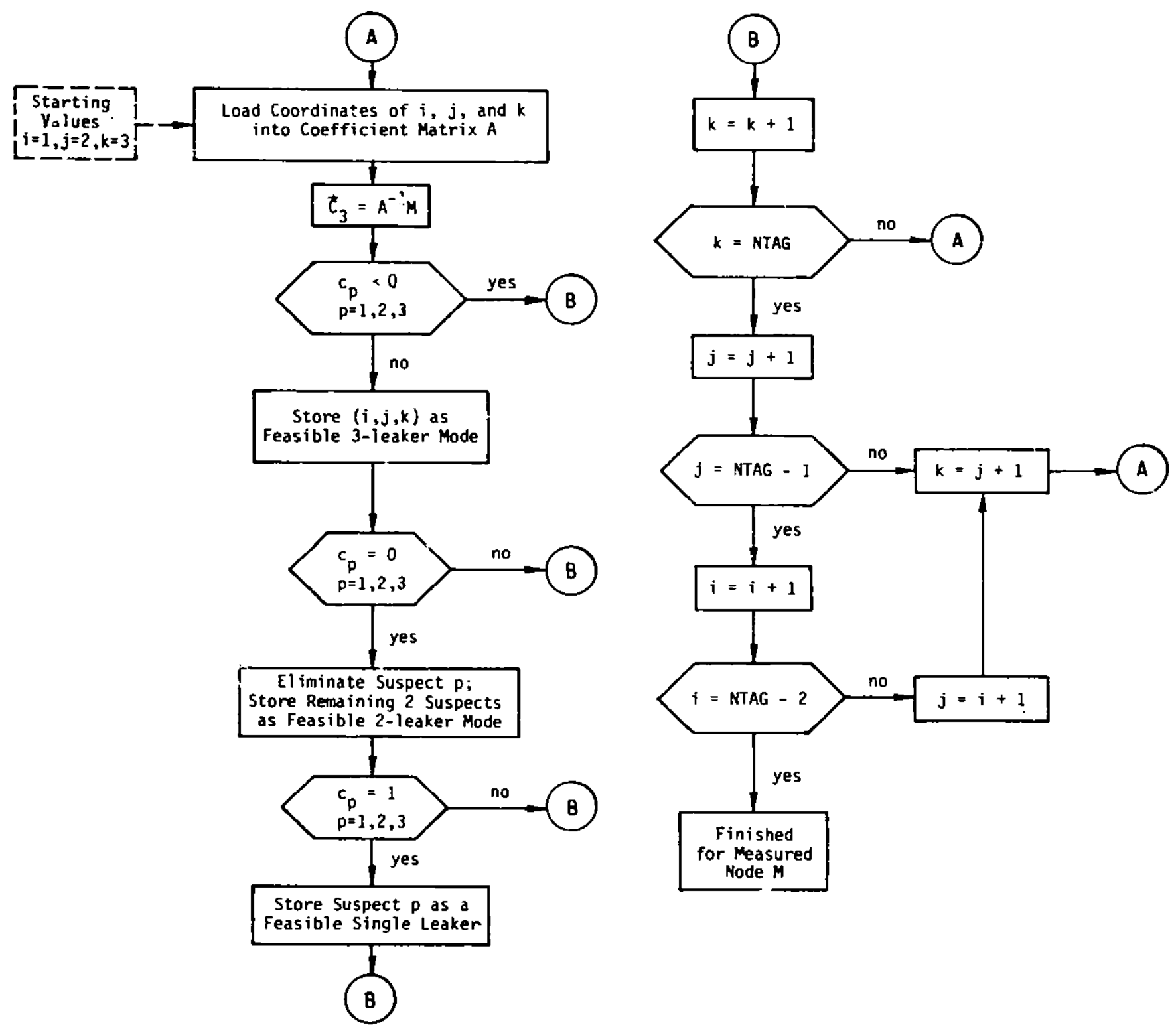

Fig. 8. Flowchart for Triangular Simplex Algoritlim 


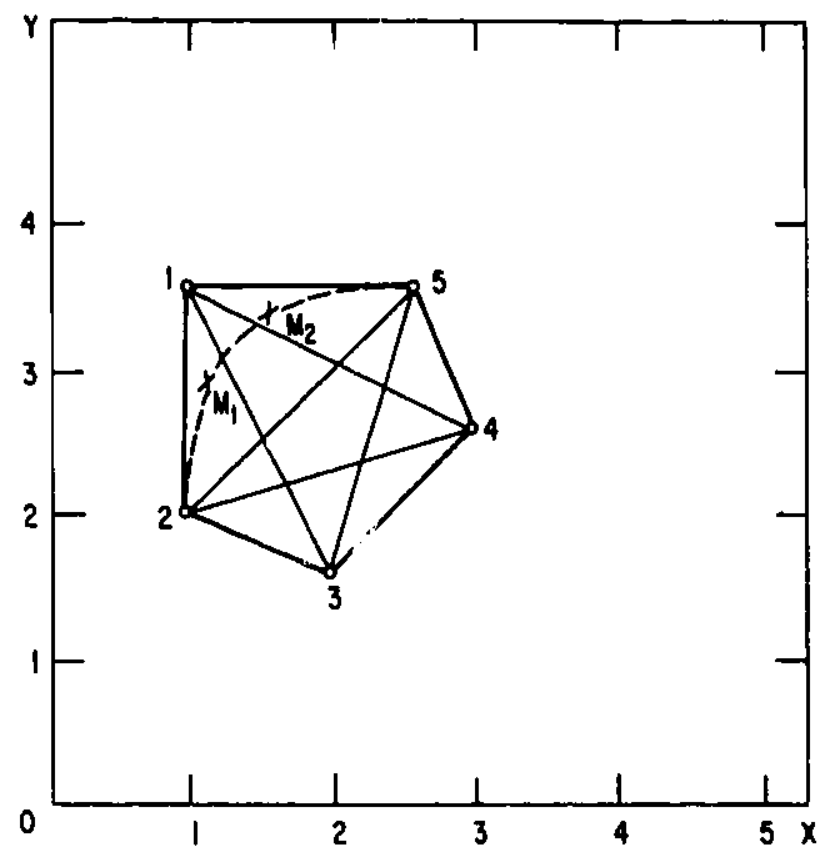

Fig. 9

Mixed-node Trajectory for Simulated Triple Failure of Assemblies 2, 1, and 5

TABLE IV. Barycentric-coordinates Technique Applied to Node $M_{1}$

\begin{tabular}{|c|c|c|}
\hline $\begin{array}{l}\text { Suapect } \\
\text { Triangle }\end{array}$ & $\begin{array}{c}\text { Teat on } \\
\text { El ement } s \text { of } \overrightarrow{\mathrm{C}}_{3}\end{array}$ & Conc lusions \\
\hline 123 & $\begin{array}{l}0<c_{1}<1 \\
0<c_{2}<1 \\
0<c_{3}<1\end{array}$ & 123 possiole mode of feilure \\
\hline 124 & $\begin{array}{l}0<c_{1}<1 \\
0<c_{2}<1 \\
0<c_{3}<1\end{array}$ & 124 possible mode of failure \\
\hline 125 & $\begin{array}{l}0<c_{1}<1 \\
0<c_{2}<1 \\
0<c_{3}<1\end{array}$ & 125 purible mode of failure \\
\hline 134 & $c_{3}<0$ & Exclude 134 from consideration \\
\hline 135 & $c_{3}<0$ & Exclude 135 from consideration \\
\hline 145 & $\mathrm{c}_{3}<0$ & Exclude 145 from consideration \\
\hline 234 & $c_{2}<0$ & Exclude $234^{\prime}$ from consideration \\
\hline 235 & $c_{2}<0$ & Exclude 235 from coneideration \\
\hline 245 & $c_{2}<0$ & Exclude 245 from consideration \\
\hline 345 & $c_{2}<0$ & Exclude 345 from consideration \\
\hline
\end{tabular}


In the first row of the table, a test is performed to determine if assemblies 1,2 , and 3 could have failed simultaneously to yield point $M_{1}$. Thus, the coordinates of nodes 1,2 , and 3 are substituted into Eq. 14 , and $\vec{C}_{3}$ is computed as described earlier. For triangle 123, it turns out that all three components of $\stackrel{+}{\mathrm{C}}_{3}$ lie between zero and one, as indicated in the second column of the table. The conclusion, shown in the third column, is that assemblies 1, 2, and 3 could have failed simultaneously to produce $M_{1}$ and therefore cannot be eliminated as suspects.

We next apply the same test to each of the other combinations of suspects listed in the first column of the table. Observe that for each of the last seven triangles considered, one of the elements of $\vec{C}_{3}$ turned out to be negative. As discussed earlier, this fact allows us to exclude each of those sets of assemblies from further consideration.

After the list of possible suspect combinations has been exhausted for point $M_{1}$, the entire procedure is repeated for the second measured node. Table $V$ gives the results for node $M_{2}$. From this table it is seen that 123,124 , and 125 are the feasible modes of failure for node $\mathrm{M}_{2}$.

TABLE V. Barycentric-coordinates Technique Applied to Node $\mathrm{M}_{2}$

\begin{tabular}{|c|c|c|}
\hline $\begin{array}{l}\text { Suspect } \\
\text { Triangle }\end{array}$ & $\begin{array}{c}\text { Test on } \\
\text { Elements of } \vec{c}_{3}\end{array}$ & Concluaione \\
\hline 123 & $c_{3}<0$ & Exclude 123 from consideration \\
\hline 124 & $c_{\boldsymbol{\jmath}}<0$ & Exclude 124 from congideration \\
\hline 125 & $\begin{array}{l}0<c_{1}<1 \\
0<c_{2}<1 \\
0<c_{3}<1\end{array}$ & 125 posaible mode of failure \\
\hline 134 & $c_{3}<0$ & Exclude 134 from consideration \\
\hline 135 & $\begin{array}{l}0<c_{1}<1 \\
0<c_{2}<1 \\
0<c_{3}<1\end{array}$ & 135 posaible mode of failure \\
\hline 145 & $\begin{array}{l}0<c_{1}<1 \\
0<c_{2}<1 \\
0<c_{3}<1\end{array}$ & 145 posaible mode of failure \\
\hline 234 & $c_{2}<0$ & Exclude 234 from coneideration \\
\hline 235 & $c_{2}<0$ & Exclude 235 from consideration \\
\hline 245 & $c_{2}<0$ & Exclude 245 from conoideration \\
\hline 345 & $c_{2}<0$ & Exclude 345 from coneideration \\
\hline
\end{tabular}

The final step is to compare the sets of feasible suspects for the two measured nodes to find a common mode of failure. For this simple example, the common mode of failure is seen to be 125 . Thus, the analysis has told us correctly that assemblies 1, 2, and 5 are all leaking. 
Note carefully that even though this example illustrated the general technique with a triple failure, the reader may satisfy himself that the same procedure would have identified a double failure had $M_{1}$ and $M_{2}$ fallen on a line connecting two tag nodes, or a single failure if they had coincided with a single tag node. In the latter case, a $c$-value of 1 for the leaking assembly would be obtained for both $M_{1}$ and $M_{2}$; in the former, a pair of complementary $c$-values ( $t$ wo values that sum to unity) would be obtained for both $M_{1}$ and $M_{2}$. It is this unique ability to identify first-, second-, or third-order failures with a series of elementary matrix inversions that brings harmony and order into what might otherwise be a hopeless chaos of experimental facts.

If $M_{1}$ and $M_{2}$ had been closer together on the mixed-node trajectory in Fig. 9 (becáuse of, say, collection of sample 2 very soon after the collection of sample 1), elimination of all the possible modes of failure may have been impossible. When this degenerate-identification problem arises in practice, the process may have to be repeated with a third and possibly a fourth measured node until a unique identification is obtained. Thus, for a reactor with a two-dimensional tag system, or one with a three-dimensional system in which many nodes 1 ie on common planes, an automatic on-line mass spectrometer with a cycling time shorter than the effective mean cleanup time for noble gases in the primary system would be essential.

C. Exploiting the Concepts of Distance in Ratio Space

The procedures described above give an idea of the barycentric-coordinates technique as applied in its simplest form. For the technique to be of maximum practical utility, however, the theory associated with it can be developed further.

Up to this point, we have described the use of analytical techniques to determine whether a given node coincides with some given point or line. In practice, however, even though the analytical techniques remain valid, there are several practical reasons why a point specified to fall on a given line may actually lie a small distance from it. The primary reason is the experimental uncertainty introduced by measurement of the tag-gas compositions.

Secondary reasons include uncertainties in the isotopic compositions caused by neutron-burnout effects.

For these reasons, it is not sufficient that the multiple-failure analysis technique tell us whether the measured node falls on the line or not on the line; it must also be capable of determining how far from the line the point lies. This applies also to situations in which we wish to know whether the point lies inside or outside a given triangle. In each of these instances, the technique would be of little practical value unless it could determine exactly how close the measured point is to the given configuration.

Fortunately, geometric properties associated with the components of $\vec{C}_{3}$ can be interpreted so that one may obtain this essential information by simply 
checking the values of each component. Figure 10 shows how the values of the barycentric coordinates are related to areas in the tag-node plane. ${ }^{4}$ In the figure, we denote by $\Delta 123$ the area of triangle 123 . In the general case for a triangle whose vertices are tag nodes $i, j$, and $k, c_{i}$ is equal to $\Delta M j k / \Delta i j k$ when $n r^{\circ}: M$ lies inside triangle $i j k$, and minus this value when $M$ lies outside the $t_{1}$ le. Note that since triangles $M j k$ and $i j k$ have a common base (i.e., line segment $j k),\left|c_{i}\right|$ is also equal to the ratio of the respective perpendicular distances of nodes $M$ and $i$ to line segment $j k$. Figures 11 and 12 illustrate this relationship for $c_{i}$ when $M$ falls inside and outside, respectively, of suspect triangle ijk.

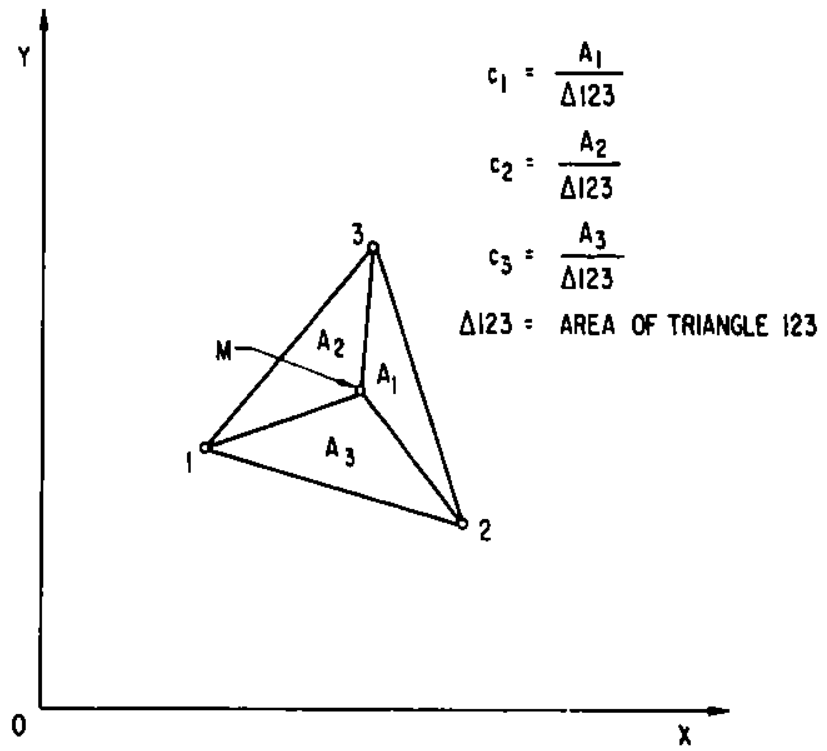

Fig. 10. Relationship of Components of $\vec{C}_{3}$ Vector to Areas in Tag..node Plane

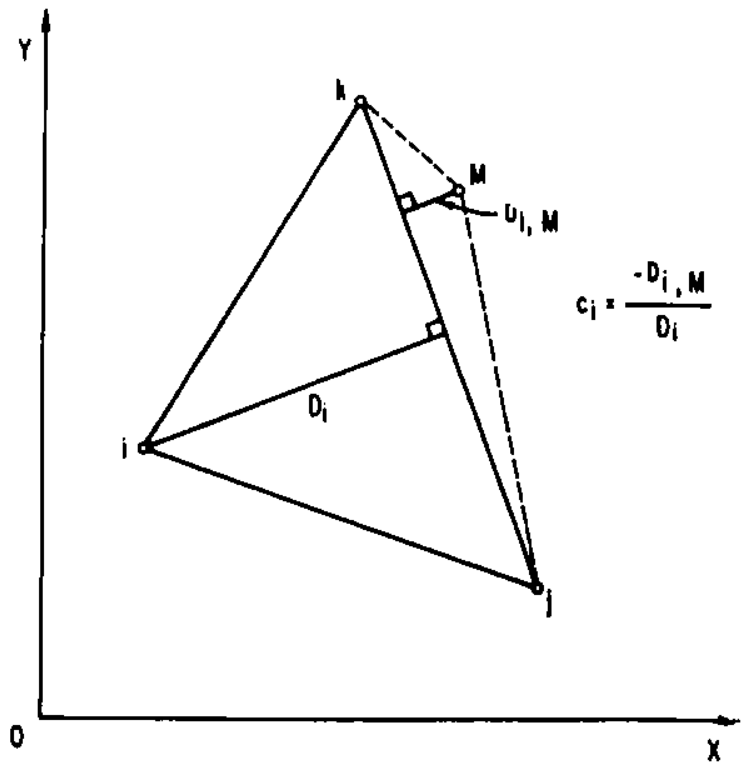

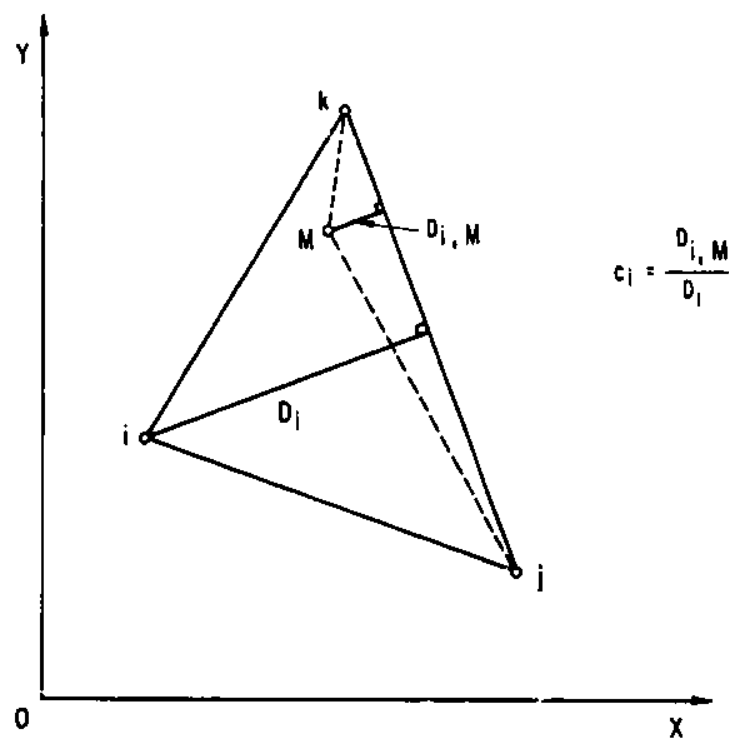

Fig. 11. Relationship between ${ }_{i}$ ind Triangle Altitudes when Measured Node Lics Inside Suspect-node Trangl?

Fig. 12

Relationsh.ip between $c_{\mathfrak{l}}$ and Triangle Altitudes when Measured Nooc Lies Outstue Suspect-node Triangle 


\section{Tetrahedral Simplex Method}

In a three-dimensional ratio space, a measured tag node can be represented uniquely by a linear combination of four noncoplanar suspect nodes. These four nodes define a tetrahedral configuration, or simplex, and the procedure developed to treat such three-dimensional problems is called the tetrahedral simplex technique. 4

The procedure already developed for two-dimensional problems can be extended immediately to three dimensions with little more than a change in notation in the governing equations. 'lo establish a transformation between the global Cartesian coordinates and the local barycentric coordinates, we begin by setting up the two equations

$$
\vec{R}=\sum_{i=1}^{4} c_{i} \vec{R}_{i}
$$

and

$$
1=\sum_{i=1}^{4} c_{i} .
$$

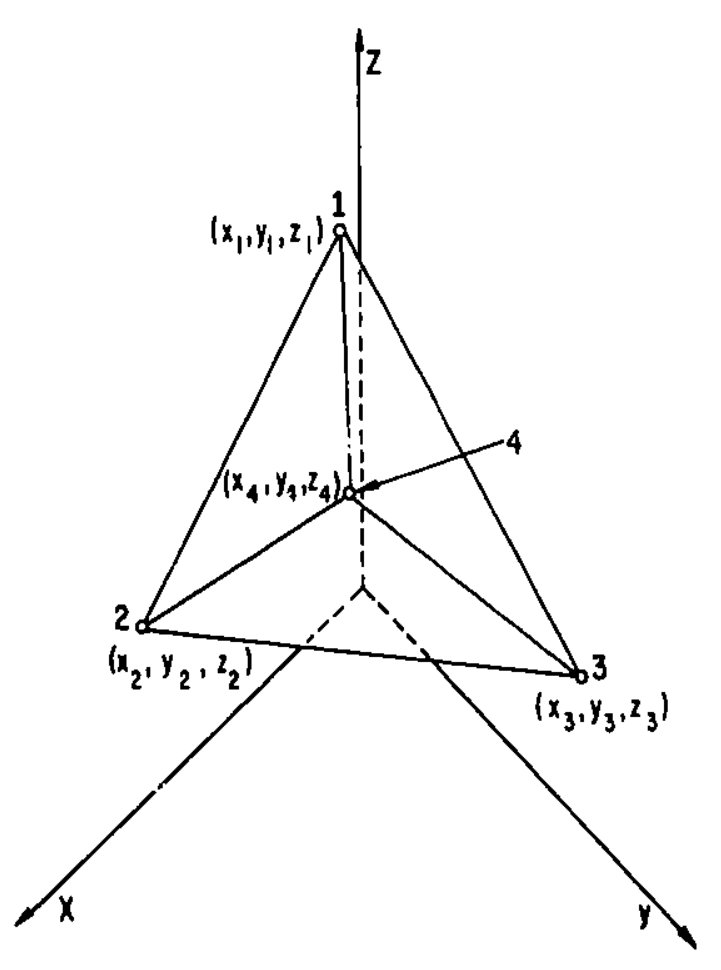

Fig. 13. Tetrahedral Node Arrangement in Three-dimensional Ratio Space
The vector $\vec{R}$ in Eq. 16 is the threecomponent vector from the origin to the measured node, and $\vec{R}_{i}$ is the corresponding vector from the origin to suspect tag node $i$. Figure 13 shows a tetrahedral node arrangement and defines the node numbering scheme. In a Cartesian system with coordinate axes $X, Y$, and $Z$, the vectors in Eq. 16 may be cast in the form

$$
\vec{R}=\left(\begin{array}{c}
x \\
y \\
z
\end{array}\right)
$$

and

$$
\vec{R}_{i}=\left(\begin{array}{c}
x_{i} \\
y_{i} \\
z_{i}
\end{array}\right)(i=1,2, \ldots, 4) \text {. }
$$

If we substitute these vectors into Eqs. 16 and 17 and express the result in matrix form, an expression is obtained that can be readily evaluated (cf. Eq. 14): 


$$
\left(\begin{array}{cccc}
x_{1} & x_{2} & x_{3} & x_{4} \\
y_{1} & y_{2} & y_{3} & y_{4} \\
z_{1} & z_{2} & z_{3} & z_{4} \\
1 & 1 & 1 & 1
\end{array}\right) \vec{c}_{4}=\left(\begin{array}{l}
x \\
y \\
z \\
1
\end{array}\right)
$$

where

$$
\overrightarrow{\mathrm{c}}_{4}=\left(\begin{array}{c}
{ }_{1} \\
\mathrm{c}_{2} \\
\mathrm{c}_{3} \\
c_{4}
\end{array}\right) .
$$

As in the two-dimensional case, geometric properties associated with the $\vec{C}_{4}$ vector permit us to identify certain suspects or combinations of suspects as "feasible" modes of failure while eliminating others from further consideration. These properties are directly analogous to the properties enunerated in Sec. III.A for the $\vec{C}_{3}$ vector.

For example, if only one assembly is leaking, the component of $\overrightarrow{\mathrm{C}}_{4}$ corresponding to that assembly will be unity, and the remaining three components will be zero. If two are leaking, the measured node will fall on the line cornecting the two corresponding tag nodes. The c's corresponding to these tro nodes will have values between 0 and 1 , and the remaining two $c$ 's will be zero. If three assemblies are leaking simultaneously, the measured node falls inside the triangle whose vertices are the three corresponding $t$ ag nodes. The three c's corresponding to these nodes will have values cetwaen 0 and 1 , and the fourth $c$ will be zero. If all four assemblies a:e leaking, the measured node falls inside the tetrahedron whuse vertices are the four corresponding tag nodes, and all four c's will have values between 0 and 1 . Finally, if the measured node falls outside the suspect tetrahedron, sne or more of the c's will be negative. In this case it means that at least one assembly other than, or possibly in addition to, the four suspects must be releasing tag gas elsewhere in the system.

Of course, when experimental uncertainties are taken into account, the computed values of the barycentric coordinates never actually equal 0 or 1 identically. Nevertheless, the linear relationship between the various $c^{\prime} s$ and distances sti'l holds in three-dimensivasl ratio space. This means that any confidence interval that can be attached to our knowledge of the location of a measured node relative to the configuration of the suspect nodes can be translated into an equivalent confidence interval for the values of the components of $\vec{C}_{4}$. 


\section{IMPORTANCE OF ACCURACY SPECIFICATION OF ASSEMBLY TAG COMPOSITIONS}

When composition uncertainties result in displacement of the measured node $M$ away from the configuration of nodes giving rise to $M$, the danger of misidentification using the barycentric-coordinates technique is small. This danger is minimized by using the best estimates of the corrected tag compositions (i.e., corrected for neutronics effects) for each assembly tag node. If the displacement between the corrected composition and the true composition of gas released from each respective leaker is small, the corresponding discrepancies in the c-values will also be small (since the magnitudes of the c-values are proportional to the relative displacements, as shown in Ser III.B).

A situation that is more likely to adversely affect the outsome of the tag analysis may arise if the position of $M$ is moved closer to a given node configuration by the effects of unrertainties. For example, suppose that, in a two-

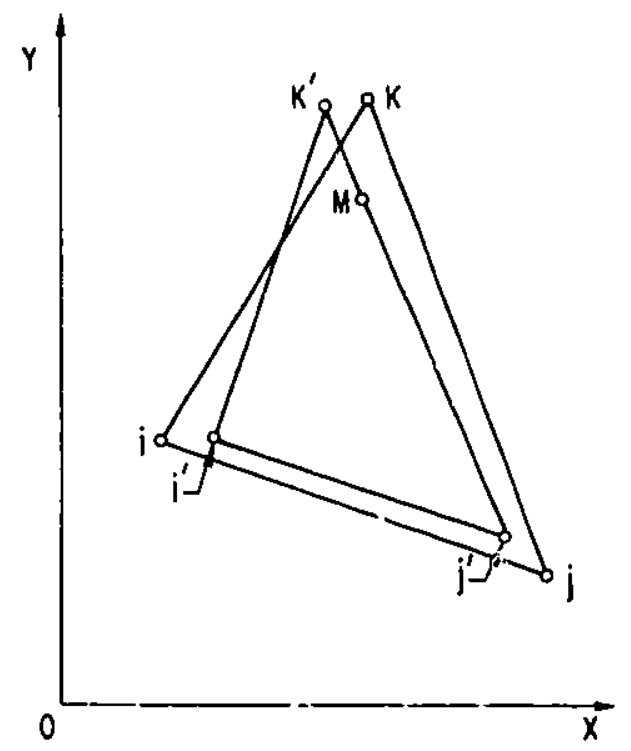

Fig. 14. Displacement of Corrected Node Positions (primed letters) from True Node Positions as a Result of Comprsition Uncertainties dimensional tag system, assemblies $i, j$, and $k$ all fail simultaneously. We know from earlier discussions that the mixture of the three released tags will give rise to a daughter $t$ ag $M$ that should fall inside triangle ijk. Consider what would happen, however, if our best estimates of the corrected tag positions are shifted from their true locations as shown in Fig. 14, in which the estimated and true positions of the three $t$ ag nodes are signified by primed and unprimed letters, respectively. The barycentric-coordinates technique, and indeed any deterministic calculational scheme that uses the coordinates of $i^{\prime}, j^{\prime}$, and $k^{\prime}$ as $i n-$ put, would incorrectly tell us that assembly $i$ is not leaking and that assemblies $j$ and $k$ are the only two leakers in the system.

This example illustrates the importance of ensuring that uncertainties in assembly tag compositions are kept as small as possible. In addition to correcting the tag compositions for neutronics effects, one can further diminish the possibility of misidentification by combining the results of the tag analysis with probability-of-failure information for the individual assemblies, using an appropriate probabilistic mode $1,16,17$

\section{TREATMENT OF BACKGROUND TAG ISOTOPES}

An additional benefit of implementation of the barycentric-coordinates technique for multiple-failure analysis is that the procedure as specified above requires no modifications to account for the presence of "background" 
noble-gas isotopes in the coolant system. One simply includes the isotopic ratios of any such gases in the file containing the assembly tag ratios. In this way, the background tags are treated as additional suspect nodes.

For example, in EBR-II's xenon-tagging system, ${ }^{3}$ two background tags are included with each set of tag nodes representing a core of assembly tags. These background tags are designated XENAT and TRAMP. Tag XENAT represents the experimentally determined composition of naturally occurring xenon that is present as a contaminant in the reactor's argon cover gas, and TRAMP represents the composition of stable fission-product xenon that is present from the fissioning of tramp uranium in the core. The isotopic ratios for these background tags are determined by mass-spectrometric analysis of argon cover-gas samples free of tag isotopes and by analysis of samples obtained during full-power operation with no leakers in the core.

Since the background tags are treated as additional suspect nodes, one does not need to know beforenand (or guess by trial and error) what proportion of a measured sample is due to release of an assembly's $t a g$ and what proportion is attributable to bankgound isotopes. Thus, for instance, if the results of a multiple-leaker analysis indicate that the most likely triple-failure combination is, say, 8-XENAT-45 in the respective percentages 25, 10, 65, interpretation would be straightforward: $10 \%$ of the measured sample is attributable to natural-xenon background isotopes; $90 \%$ is attributable to tag isotopes released from assemblies 8 and 45 , with $28 \%$ [i.e., $25 /(25+65)$ ] coming from assembly 8 and $72 \%$ coming from assemb?y 45 .

Examples illusirating the identification of released assembly tags in the presence of background roble-gas isotopes are presented later in Sec. VIII.

\section{SUSPECT-RANKING ALGORITHMS}

POLYFAIL determines all possible modes of failure, using the tetrahedral simplex method outlined in Sec. III. The code then transfers control to subroutine RANKER, which ranks the possible failure modes according to the value of a distance parameter, $H$. For one-leaker suspects, $H$ is the Fuclidian distance in three-dimensional ratio space from the measured tag node to the suspect node. Physically, $\mathrm{H}$ is simply the root-mean-square deviation between the isotopic ratios for the measured and suspect tags and is determined by

$$
H=\left[\sum_{i=1}^{3}\left(M_{i}-s_{i}\right)^{2}\right]^{1 / 2} \text {. }
$$

In this equation, $M_{i}$ and $S_{i}$ are the component ratios for the measured and auspect nodes, respectively.

For the various possible two- and three-leaker modes of failure, the suspect combinations are ranked using algorithms developed in the following sections. It will be assumed throughout that the suspect tag compositions have been corrected for isotopic production and depletion effects. 


\section{A. Two-leaker Combinations}

Four ranking algorithms have been incorporated into subroutine RANKER for sorting the two-leaker combinations. Each algorithm ranks the suspect combinations according to different sets of criteria as described in the following paragraphs. The selection of the most appropriate algorithm to use for a given POLYFAIL run is left as an input option that is specigied by the user at the time of execution.

Suppose a measured $t a g ~ M$ is obtained by mass-spectrometric analysis of a sample of reactor cover gas that nay contain a nonnegligible quantity of background tag gas B. For example, gas B could comprise residual isotopes remaining in the cover gas from one or more previously released tags, isotopes of naturally occurring noble gas present $: 3$ a contaminant in the cover gas, or fission-produced isotopes present from fissioning of tramp fuel in the core. The original algorithm devised to determine which assembly is most likely to be the source of the most recent tag release consists of computing a distance parameter $\mathrm{H}_{i}$ for each assembly tag, $S_{i}$, where $i$ ranges from 1 to the number NTAG of distinct tags in the system. 18 In a three-dimensional tag-ratio space, the axes of which represent the independent ratios of the mole fractions of the characteristic tag isotopes, $H$ is the perpendicular distance from node $M$ to vector $\vec{V}_{S}$, where $\vec{V}_{S}$ connects background node $B$ with suspect node $S_{i}$ (see case a, Fig. 15)

(0)

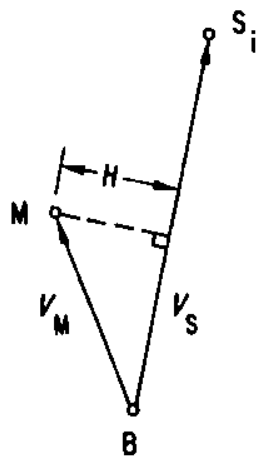

(c)

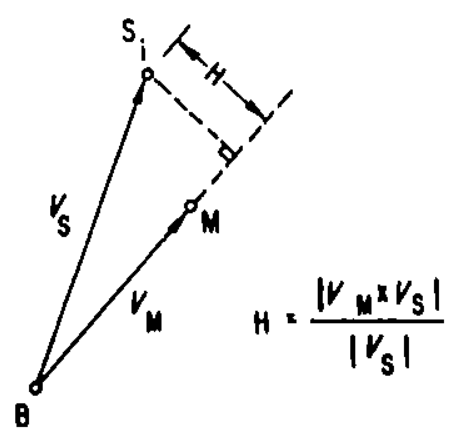

(b)

$$
H=\frac{\left|V_{M} \times V_{S}\right|}{\left|V_{S}\right|}
$$

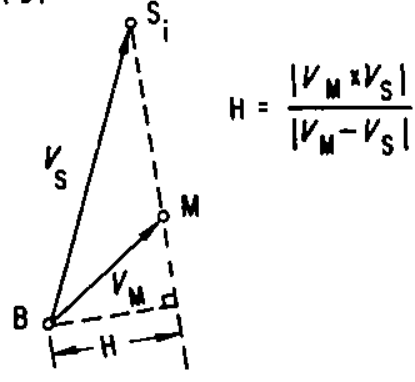

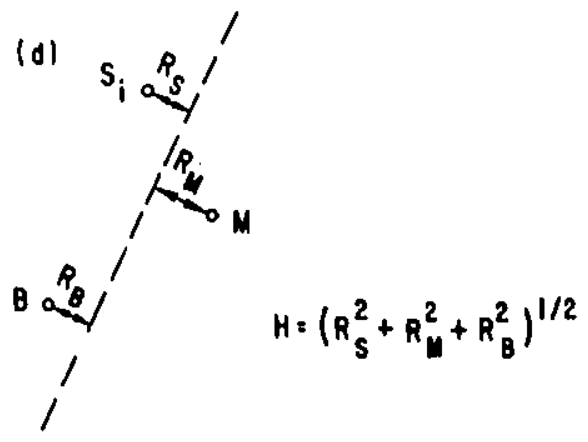

Fig. 15. Alternative Ranking Algorithms for Resolution of Double Element Failures 
Application of the foregoing algorithm implicitly assumes that the locations of $t a g$ nodes $B$ and $S_{i}$ are known accurately, but allows for uncertainties in the coordinates of $M$ that arise from experimental errors in massspectrometric analysis. Experience with such analysis of mixed-tag samples has shown that situations may arise, especially during operation with higher-burnup fuel, in which the uncertainties in the background composition or the suspect compositions may be greater than the uncertainty in the measured $t a g$. Three such situations are addressed here. The algorithms devised to treat trese situations are illustrated by cases b, c, and d in Fig. 15.

The situation that arises most frequently is one in which the uncertainties in the composition of the background tag outweigh the uncertainties in either $S_{i}$ or $M$. This situation is a result of tr a relatively small concentrations of background isotopes in the cover gas. In this case, the most appropriate definition of $\mathrm{H}$ is that depicted in case b of Fig. 15. This definition provides a measure of the deviation of $B$ from the straight line passing through $\mathrm{S}_{\mathrm{i}}$ and $\mathrm{M}$.

During operation with high-burnup fuel, a situation may arise in which one has confidence in the compositions of both $B$ and $M$, but the assembly tags have relatively large uncertainties due to neutron burnout. In this case, one would rank the suspect tags using the definition of $\mathrm{H}$ depicted in case $c$ of $\mathrm{Fig} .15$. This definition of $\mathrm{H}$ measures the deviation of $S_{i}$ from the straight line passing through $B$ and $M$.

Finally, for situations in which one has equal confidence in the compositions of $S_{i}, B$, and $M$, the most appropriate definition of $H$ is that depicted in case $d$ of Fig. 15, which assumes that the relative uncertainties in $B, S_{i}$, and $M$ are equal. In this case, $H$ provides a measure of the root mean square of the deviations of $B, S_{i}$, and $M$ from the best-fit line passing through the three nodes.

Routines for all four algorithms have been incorporated into subroutine RANKER. When no information is available on the relative uncertainties of the suspect, background, and measured tag comprsitions, or when those uncertainties are estimated to be equivalent, the routine corresponding to case $d$ is used by default for ranking the most likely two-leaker analyses.

\section{B. Three-1eaker Combinations}

When three assemblies are releasing tag gas simultaneously, we know from considerations presented in Sec. II that the measured tag node should be in the plane containing the three tag nodes and, moreover, should be confined to the triangle whose sides connect those three nodes. In practice, however, because of the various uncertainties described in Sec. III.B, the measured node will seldom be found to be exactly in the triangle plane. The perpendicular distance of the measured node to the suspect-triangle plane therefore providcs a suitable quantitative ranking indicator that can be used to rank the most likely three-leaker suspects. 
We denote by $\vec{R}_{i}, \vec{R}_{j}, \vec{R}_{k}$, and $R_{M}$ the vectors from the origin to the respective tag nodes $i, j, k$, and $M$. The distance parameter $H i s$ defined to be the magnitude of the vector $\vec{H}$, where $\vec{H}$ is evaluated from

$$
\vec{I}=\frac{(\vec{M}-\vec{J}) \cdot[(\vec{K}-\vec{J}) \times(\vec{I}-\vec{J})]}{|(\vec{K}-\vec{J}) \times(\vec{I}-\vec{J})|} .
$$

In this expression, vertical bars signify the magnitude of the denominator vector, and - and $x$ signify the dot and cross products, 19 respectively.

C. Four-leaker Combinations

In a three-dimensional ratio space, four-leaker modes of failure cannot be ranked on the basis of any results from the tag analyses. Either a suspect tetrahedron encloses the measured tag node or it does not. All suspect tetrahedra enclosing the measured tag node must be considered equally likely modes of failure. POLYFALL identifies all such tetrahedra in accordance with the criteria established in Sec. III.D, flags them as feasible modes of failure, and stores their associated assembly identification numbers (ID's) for subseuent retrieval and oucput.

\section{I. CALCULATIONAL STRATEGIES}

We begin this section with a brief discussion of the theory associated with the linear independence of ratio space. It will be shown that limitations imposed by considerations of linear independence completely fix the maximum number of tagged assemblies that can be resolved with POLYFAIL. The remainder of the section is devoted to the development of analytical methods that have been incorporated into POLYFAIL to reduce complexity and computational effort.

\section{A. Limitations Imposed by Considerations of Linear Independence}

In an $\mathrm{N}$-dimensional tag opace, any one combination of at most $\mathrm{N}+1$ assembly tags can, in principle, be identified. Thus, in a two-dimensional tag space, there are at most a finite number of distinct combinations of three or less assembly tags that could have failed to produce any given measured tag. Moreover, by use of a systematic mathematical procedure (such as the barycentric-coordinates technique), each of those combinations can be identified and listed.

However, a finite number of feasible fourth-order modes of failure could not be identified in a two-dimensional tag opace. The reason, mathematically, atems from the fact that any four nodes that lie in a common plane are linearly dependent, 20 and therefore a general measured node cannot be expressed as a unique linear combination of those four nodes. 
This concept of linear dependence is a general one and extends to higher and lower dimensional node arrangements as well. For a one-dimensional arrangement in which several nodes may lie on a common 1 ine, the maximum number of nodes that could be tested for feasibility would be two. Triple-learer analyses would be impossible. (Historically, the earliest tag design put into practice used one-dimensional node arrangements.) ${ }^{l}$

For a three-dimensional system of tags (i.e., those which POLYFAIL was developed to address), the maximum number of nodes that can be tested as a feasible mode of failure is four. Thus, as we have seen in Sec. III, POLYFAIL breaks up the system of tags into all possible distinct permutations of nodes taken four at a time. It then tests each of those sets of nodes for feasibility, using the techniques in roduced in S.C. III.D. If, however, it encounters a set of four nodes that are coplanar, the linear-independence criterion dictates that those four nodes cannot be tested as a feasible four-leaker combination. In fact, if an attempt were made to apply the tetrahedral simplex technique to a set of four coplanar nodes, Eq. 20 would be inconsistent, and incersion of the matrix in that equation would produce overflow errors.

A simple test for linear independence that avoids this difficulty is developed in Sec. VII.B below. Any combination of nodes that are determined to be coplanar (or nearly coplanar) are still tested in subsets for feasible triple-, double-, or single-leaker modes of failure.

It is important to point out that the foregoing restrictions on the maximum number of simultateous leakers that can be identified with the POLYFAIL code are by no means unique to the barycentric-coordinates method of solution. The theoretical 1 imit on the number of simultaneous failures that can be resolved is completely fixed by the dimensionality of the assembly-node arrangement. As a practical matter, however, these restrictions are not severe, because for each three-dimensional tag system proposed to date, $3,4,21,22$ the probability is remote of having a simultaneous release of tags from four assemblies whose tags happen to be coplanar.

\section{B. Analytical Test for Coplanar Node Arrangements}

To avoid the difficulties just described, a simple and extremely efficient analytical test has been devised to identify any sets of coplanar tag nodes before their coordinates are passed to the tetrahedral simplex algorithm. The test makes use of the determinant form of the equation for the volune $V$ of a tetrahedron whose vertices are represented by the ordered triples $\left(x_{i}, y_{i}, z_{i}\right)$, $1<i<4$. From Ref. 23 this equation is

$$
v=(1 / 6)\left|\begin{array}{llll}
x_{1} & x_{2} & x_{3} & x_{4} \\
y_{1} & y_{2} & y_{3} & y_{4} \\
z_{1} & z_{2} & z_{3} & z_{4} \\
1 & 1 & 1 & 1
\end{array}\right| .
$$


(The use of vertical bars in the notation for a determinant and in the notation for the magnitude of a vector, while perhaps unfortunate, is universal. Which meaning is intended in any part cular case should be clear from the context.)

For any four tag nodes that $l i e$ in a common plane, $v$ will vanish. Thus, to ensure that four suspect tag nodes are noncoplanar, we can require that

$$
\left|\begin{array}{llll}
x_{1} & x_{2} & x_{3} & x_{4} \\
y_{1} & y_{2} & y_{3} & y_{4} \\
z_{1} & z_{2} & z_{3} & z_{4} \\
1 & 1 & 1 & 1
\end{array}\right|>\varepsilon
$$

where $E$ is a nonnegative scalar constant chosen to be sufficiently jarge that overflow errors will be avoided in solution of matrix Eq. 20 .

The efficiency with which Eq. 25 can be implemented can be further enhanced by application of elementary column transformations. ${ }^{4}$ For example, if we add $(-1)$ times the elements of column 1 to the corresponding elements of columns 2,3 , and 4 , we obtain the new but equal determinant equation

$$
\left|\begin{array}{cccc}
x_{1} & x_{2}-x_{1} & x_{3}-x_{1} & x_{4}-x_{1} \\
y_{1} & y_{2}-y_{1} & y_{3}-y_{1} & y_{4}-y_{1} \\
z_{1} & z_{2}-z_{1} & z_{3}-z_{1} & z_{4}-z_{1} \\
1 & 0 & 0 & 0
\end{array}\right|>\varepsilon .
$$

By application of Laplace's expansion 25 in terms of the fourth row, we obtain the more simplified expression

$$
\left|\begin{array}{lll}
x_{2}-x_{1} & x_{3}-x_{1} & x_{4}-x_{1} \\
y_{2}-y_{1} & y_{3}-y_{1} & y_{4}-y_{1} \\
z_{2}-z_{1} & z_{3}-z_{1} & z_{4}-z_{1}
\end{array}\right|<-\varepsilon .
$$

Note that the negative sign associated with the row -4 , column-1 cofactor has been absorbed in this last equation by multiplying through by $(-1)$ and reversing the sense of the inequality.

Experience gained by applying POLYFAIL to EBR-II's tag analyses has shown that overflow errors can be avoided in single-precision calculations by specifying a value of $\varepsilon=1.0 \times 10^{-4}$.

\section{Matrix-inversion Calculationa}

The logical structure of the POLYFAIL code as outlined in Sec. III.C is relatively simple. Most of the computational effort required by the code is 
devoted to the large number of repetitions of the matrix-inversion step in the solution of Eq. 20. To maximize the efficiency of the code, some methods that can be usid to reduce complexity and computational effort in the matrixinversion process are developed here.

A solution to Eq. 20 could be obtained by direct application of Cramer's rule. ${ }^{24}$ However, by taking advantage of the special form of the matrices involved, we can make a relatively simple coordinate transformation that results in an appreciable reduction in computational effort. We begin by premultiplying both sides of Eq. 20 by the inverse of the coefficient matrix. The result is

$$
\vec{c}_{4}=\left(\begin{array}{cccc}
x_{1} & x_{2} & x_{3} & x_{4} \\
y_{1} & y_{2} & y_{3} & y_{4} \\
z_{1} & z_{2} & z_{3} & z_{4} \\
1 & 1 & 1 & 1
\end{array}\right)^{-1}\left(\begin{array}{l}
x \\
y \\
z \\
1
\end{array}\right) .
$$

An important characteristic of the global Cartesian-coordinate system is its invariance with respect to linear translation. 26 we can take advantage of this fundamental property by displacing the components of each tag node in the syst $t m$ in such a way that the resulting (translated) coordinate system has as its origin the measured $t a g ~ M$. If the new coordinates are signified by primes, Eq. 28 becomes

$$
\vec{C}_{4}=\left(\begin{array}{rrrr}
x_{1}^{\prime} & x_{2}^{\prime} & x_{3}^{\prime} & x_{4}^{\prime} \\
y_{1}^{\prime} & y_{2}^{\prime} & y_{3}^{\prime} & y_{4}^{\prime} \\
z_{1}^{\prime} & z_{2}^{\prime} & z_{3}^{\prime} & z_{4}^{\prime} \\
1 & 1 & 1 & 1
\end{array}\right)^{-1}\left(\begin{array}{l}
0 \\
0 \\
0 \\
1
\end{array}\right) .
$$

If we denote by ||$A||$ the inverted matrix on the right (where double vertical bars are used to distinguish the matrix from its determinant) then we can write

$$
\stackrel{C}{C}_{4}=\| A \mid\left(\begin{array}{l}
0 \\
0 \\
0 \\
1
\end{array}\right) \text {. }
$$

Following the elementary rules of matrix multiplication, we obtain the extremely useful result

$$
c_{1}=\left.|| A\right|_{1,4}, c_{2}=\left.|| A\right|_{2,4}, c_{3}=\left.|| A\right|_{3,4}, c_{4}=\left.|| A\right|_{4,4}
$$


In words, Eq. 31 states that the components of the solution vector $\vec{C}_{4}$ are supplied directly by the elements occupying the fourth column of the inverted coefficient matrix.

D. Incorporation of Ratio-weighting Factors for Optimized Sersitivity

We develop here a ratio-weighting scheme that $c$ an be applied to normalize the components of the assembly tag ratios in such a way that the overall sensitivity of the tag-analysis procedure is optimized. Application of the ratioweighting scheme is desirable for the foilowing reasons.

1. The component tag ratios used to characterize the system of tags may have values that fall in vastly different ranges of magnitude. For example, LoR-II's tagging system is characterized by the ratios $\mathrm{Rl}={ }^{126} \mathrm{Xe} /{ }^{124} \mathrm{Xe}, \mathrm{R} 2=$ ${ }^{128} \mathrm{Xe} /{ }^{124} \mathrm{Xe}$, and $\mathrm{R} 3=129 \mathrm{Xe} / 124 \mathrm{Xe}$. Values of these ratios typically fall in the fo:lowing ranges: $R 1=0.3-0.9, R 2=1-40$, and $R 3=10-90$. Clearly, an attempt to sum together squares of numbers of such disparate orders of magnitude would be undesirable; the residuals of the largest ratios (i.e., R3) would completely dominate those of Rl, and any information coltained in the latter would be lost.

2. For some tag analyses, one of the teg ratios may be known to be less reliable than the others, and we want to make sure our parameter estimates will be less influenced by those than by the more accurate ones.

The solution to both of these problems is obtained by a variation of the we11-known method of weighted least squares. 27,28 Briefly, the procedure is to assign a nonnegative weight factor to the three principal isotopic ratios for each tag in the system with a model ${ }^{29}$ that takes the general functional form

$$
\stackrel{+}{S}_{\text {new }}^{i}=K \stackrel{+}{s}_{o l d}^{i}|| w||,
$$

where $\vec{s}^{i}$ is the vector from the origin to the suspect tag node $i, K$ is an arbitrary normalization constant (asaigned a value of 100 in POLYFAIL), an ||$|w| \mid$ is a special $3 \times 3$ diagonal matrix. The elements of ||$w||$ are given by

$$
|| w \|_{j, h}=w_{j} / m_{j} \text { for } j=h, 1<j<3,1<h<3 \text {, }
$$

and

$$
\left.|| w\right|_{j, h}=0 \text { tor } j \neq h .
$$

In these expressions, $m_{i}, i=1,2,3$, are the original isotopic ratios of tha measured tag node, and $w_{i}, i=1,2,3$, are dimensionless ratio weighting factors. 
The final step of the procedure is to assign the following values to the measured tag iatios:

$$
m_{j, \text { new }}=\mathrm{Kw}_{\mathrm{j}}, \mathrm{j}=1,2,3 .
$$

The weighting factors, which are input at $t ;$ me of execution, can be assigned values between 0 and 1 : thereby enabling the user to selectively "tune" the analysis according to the relative degree of uncertainty associated with each of the three principal ratios. Thus, if there is equal confidence in the values of all three ratios, the weighting factors can be set equal to 1 . This ensures that informaticn from all three ratios will contribute equally in the determination of the most likely mode of failure. Alternatively, if one or more of the ratios have 1 high degree of uncertainty, the corresponding weighting factors can be specified to be less than 1, which will cause those ratios to be selectively deemphasized in all subsequent POLYFAIL calculations.

\section{E. Preservation of Linearity}

The various coordinate-transformation operations introduced in Secs. VII.C and VII.D above enhance both the efficiency and the overall sensitivity of the multiple-leaker-analysis procedures used in POLYFAIL. Before the coordinatetransformation operations can be implemented, however, one must ensure that the linearity of tag space is not compromised by afplication of those operations. The leaker-analysis procedures rely, for txampie, on the principle that, when two elements fail, the composition of the mixture lies on the line connecting the two tags of the leakers. We must therefore be certain that this relationship will continue to hold after the coordinate-transformation operations are applied. Likewise, we must ensure that, when three elements fail, the mixedtag node will lie in the triangle whose sides connect the three tag nodes. These analytical properties are of fundamental importance insofar as they are exploited in the resolution of multiple failures.

Preservation of linearity is guaranteed for the coordinate-translation operations of Sec. VII.C by the property of spatial isotropy, 30 also known as the postulate of free mobility. 31 Preservation of linearity for the coordinate-weighting scheme has been established by a rigorous mathematical proof. Detalls of the proof are straightforward, but somewhat tedious, and have been relegated to Appendix A. 


\section{SAMPLE PROBLEMS}

Four sample problems are provided to demonstrate operation of POLYFAIL. The reference system of tag nodes used is that designed for the first two cores of the FFTF. 22 Included are 80 tags designed for the FFTF driver assemblies, and 20 for the control assemblies. Each of these 100 tags is characterized by the three independent ratios of $78 \mathrm{Kr} / 80 \mathrm{Kr}, 82 \mathrm{Kr} / 80 \mathrm{Kr}$, and $126 \mathrm{e} / 129 \mathrm{dt}$ For simplicity, the tags have been assigned ordinal numbers from 1 to 100 , which will serve also as the assembly ID numbers in each example considered. A final tag having the composition $78_{\mathrm{Kr}} / 80 \mathrm{Kr}=0.1565,82 \mathrm{Kr} / 80_{\mathrm{Kr}}=5.16$, and $126 \mathrm{Kr} /$ $129 \mathrm{Kr}=0.00346$, and bearing $\mathrm{tag}$ ID 101 , has been added to the reference system to represent a typical "background" tag (see Sec. V). Appendix B provides a complete listing and a plot of the reference system of tag nodes.

In all examples, the $t a g$ ratios have been normalized with weighting factors of $\left(w_{1}, w_{2}, w_{3}\right)=(1,1,1)$ (recall Sec. VII.D). Also, in problems involving two-leaker situations, the ranking algorithm corresponding to case a in Fig. 14 (see Sec. VI.A) has been selected.

\section{A. Example 1}

As a first example, we consider the simplest case possible, that involving a single tag release with no background contamination and negligible composition uncertainties. To simulate this idealized siturtion, we arbitrarily assume that an elenent in assembly 60 has released its tag gas. Accordingly, we input the composition of $t a g$ as the measured $t a g$, or $M=(0.5909,3.290$, 0.01835). Table VI lists the five most likely (best) suspects as determined

TABLE VI. Best Five One-leaker Combinations for Measured Node $M$ in Example 1

\begin{tabular}{clr}
\hline Ranking & $\begin{array}{l}\text { Tag } \\
\text { ID }\end{array}$ & \multicolumn{1}{c}{ H } \\
\hline 1 & 60 & 0.0 \\
2 & 59 & 20.3 \\
3 & 73 & 21.6 \\
4 & 36 & 27.7 \\
5 & 55 & 28.5 \\
\hline
\end{tabular}
by POLYFAIL. The column of compited $\mathrm{H}$ values contains the geometric distance of each suspect node to the measured node $M_{1}$. (See Sec. VI for a discussion of the uses of $\mathrm{H}$ as a suspect-ranking parameter.) As expected, assembly 60 is the top suspect with a "perfect-fit" H-value of zero. For this idealized situation, at least, positive identification of the correct leaker would be assured.

\section{B. Example 2}

This example considers a one-leaker problem similar to the one just presented, except that here we assume that the various experimental uncertainties outlined in Sec. II.B combine to effect a displacement of $5 \%$ in the location of the measured node $M$ from its true location. To simulate this situation, we shift each of the component ratios for the measured tag used in Example 1 by $5 \%$ of their nominal values. The coordinates of this displaced tag are given by $M=(0.6204$, $3.455,0.01927)$. 
Application of POLYFAIL to this problem produces the results summarized in Tables VII and VIII for the most $1 \mathrm{ikely}$ one- and two-leaker combinations, respectively. The effects of the simulated experimental uncertainties are reflected in the increase to 8.3 in the value of the distance parameter associated with assembly 60 in Table VII. Nevertheless, assembly 60 is still the best suspect, and the chances of misidentification under the circumstances assumed in this example remain small.

TABLE VII. Best Five One-leaker Suspects for Example 2

\begin{tabular}{clr}
\hline Ranking & $\begin{array}{l}\text { Tag } \\
\text { ID }\end{array}$ & H \\
\hline 1 & 60 & 8.3 \\
2 & 73 & 25.0 \\
3 & 59 & 25.0 \\
4 & 36 & 28.5 \\
5 & 55 & 34.6 \\
\hline
\end{tabular}

\section{Example 3}

In this example, we illustrate application of POLYFAIL to a simulated multiple-failure case involving the simultaneous release of tags from assemblies 13 and 32. Recall from Sec. II that, for a two-tag release, the mixed-node trajectory lies along the line connecting the nodes of the two assemblies in ratio space. For this example, we have selected two points on the line conrecting tag nodes 13 and 32. We will let these points represent two possible measured nodes that might be obtained following simultaneous failure of assemblies 13 and 32. These measured nodes are labeled $M 1$ and M2. Figure 16 depicts the relative locations of $\mathrm{Ml}$ and $\mathrm{M} 2$ along the simulated two-leaker trajectory. The coordinates of $\mathrm{Ml}$ and M2 are

$M 1=(0.27595,3.6680,0.014385)$

and 
Tables IX-XI list the rost likely single-, double-, and triple-leaker combinations as determined by POLYFAIL for node Ml. In addition to those results, POLYFAIL identified $\mathrm{N} 4=26521$ feasible four-leaker modes of failure. This means that, if one were to consider the unlikely possibility of four tags being in the system at the same time, he would have 26521 equally likely combinations of assemblies that could have failed to produce measured node $\mathrm{Ml}$.

\begin{tabular}{ccc}
$\begin{array}{c}\text { TABLE IX. Best Five One- } \\
\text { leaker Suspects for } \\
\text { Measured Node Ml } \\
\text { in Example } 3\end{array}$ \\
\hline & Tap & \\
Ranking & ID & H \\
\hline 1 & 27 & 7.9 \\
2 & 26 & 18.7 \\
3 & 19 & 20.2 \\
4 & 31 & 22.5 \\
5 & 18 & 24.0 \\
\hline
\end{tabular}

$\begin{gathered}\text { TABLE } X \text {. Best Ten Two-leaker } \\
\text { Combinations for Measured } \\
\text { Node Ml in Example }\end{gathered}$
\begin{tabular}{crcc} 
Ranking & Tag & ID ' & H \\
\hline 1 & 13 & 32 & 0.0 \\
2 & 19 & 34 & 0.9 \\
3 & 14 & 52 & 1.1 \\
4 & 3 & 58 & 1.3 \\
3 & 2 & 27 & 1.5 \\
6 & 16 & 29 & 1.9 \\
7 & 11 & 90 & 2.1 \\
8 & 3 & 27 & 2.1 \\
9 & 19 & 57 & 2.2 \\
10 & 25 & 81 & 2.4 \\
\hline
\end{tabular}

\begin{tabular}{|c|c|c|c|c|}
\hline \multirow{2}{*}{$\frac{\text { Ranking }}{1}$} & \multicolumn{3}{|c|}{ Tag ID's } & \multirow{2}{*}{$\frac{H}{0.003}$} \\
\hline & 12 & 24 & 43 & \\
\hline 2 & 18 & 23 & 84 & 0.003 \\
\hline 3 & 16 & 17 & 80 & 0.005 \\
\hline 4 & 19 & 30 & 34 & 0.009 \\
\hline 5 & 18 & 21 & 28 & 0.010 \\
\hline 6 & 7 & 76 & 91 & 0.011 \\
\hline 7 & 19 & 24 & 26 & 0.012 \\
\hline 8 & 10 & 16 & 79 & 0.016 \\
\hline 9 & 19 & 23 & 38 & 0.019 \\
\hline 10 & 54 & 85 & 99 & 0.032 \\
\hline
\end{tabular}

Tables XII-XIV summarize the POLYFAIL results for measured node M2. The number of feasible four-leaker modes of failure for node $M 2$ was determined to be $\mathrm{N}_{4}=18033$.

\begin{tabular}{|c|c|c|}
\hline Ranking & $\begin{array}{l}\text { Tag } \\
\text { ID }\end{array}$ & H \\
\hline 1 & 31 & 16.5 \\
\hline 2 & 27 & 17.0 \\
\hline 3 & 28 & 20.8 \\
\hline 4 & 19 & 20.9 \\
\hline 5 & 32 & 22.7 \\
\hline
\end{tabular}

\begin{tabular}{|c|c|c|c|}
\hline Ranking & Tag & ID's & H \\
\hline 1 & 13 & 32 & 0.0 \\
\hline 2 & 12 & 47 & 0.8 \\
\hline 3 & 28 & 41 & 0.9 \\
\hline 4 & 4 & 69 & 1.2 \\
\hline 5 & 19 & 64 & 1.4 \\
\hline 6 & 29 & 82 & 1.4 \\
\hline 7 & 22 & 44 & 1.5 \\
\hline B & 20 & 61 & 1.8 \\
\hline 9 & 10 & 48 & 1.9 \\
\hline 10 & 3 & 72 & 1.9 \\
\hline
\end{tabular}

\begin{tabular}{|c|c|c|c|c|}
\hline \multirow{2}{*}{$\frac{\text { Ranking }}{1}$} & \multicolumn{3}{|c|}{ Tag ID'B } & \multirow{2}{*}{$\frac{H}{0.001}$} \\
\hline & 19 & 34 & 48 & \\
\hline 2 & 8 & so & 64 & 0.001 \\
\hline 3 & 14 & 44 & 71 & 0.002 \\
\hline 4 & 4 & 56 & 61 & 0.003 \\
\hline 5 & 10 & 20 & 79 & 0.003 \\
\hline 6 & 1 & 4 & 70 & 0.005 \\
\hline 7 & 4 & 24 & 77 & 0.009 \\
\hline 8 & 23 & 28 & 42 & 0.011 \\
\hline 9 & 3 & 34 & 48 & 0.011 \\
\hline 10 & 10 & 32 & 54 & 0.014 \\
\hline
\end{tabular}

As a final step of the analysis, POLYFAIL compares the feasible modes of failure for measured nodes $M 1$ and $M 2$ to identify any common nodes of failure. Table XV gives the results of this comparison. The first row of the table lists the number of common one-leaker modes of failure, which is zero for this example. The second row of the table shows that the code identified one common two-leaker mode of failure. The code then prints out that mode of failure, giving its computed distance from both $M 1$ and $M 2$. In the final two rows of the table, we find that the code identified no common three-leaker modes of failure and 586 common four-leaker modes of failure. (The option to print the identities of the four-leaker modes was not invoked for this example.) 
TABLE XV. Common Modes of Failure for Measured Tags $M 1$ and $M 2$ in Example 3

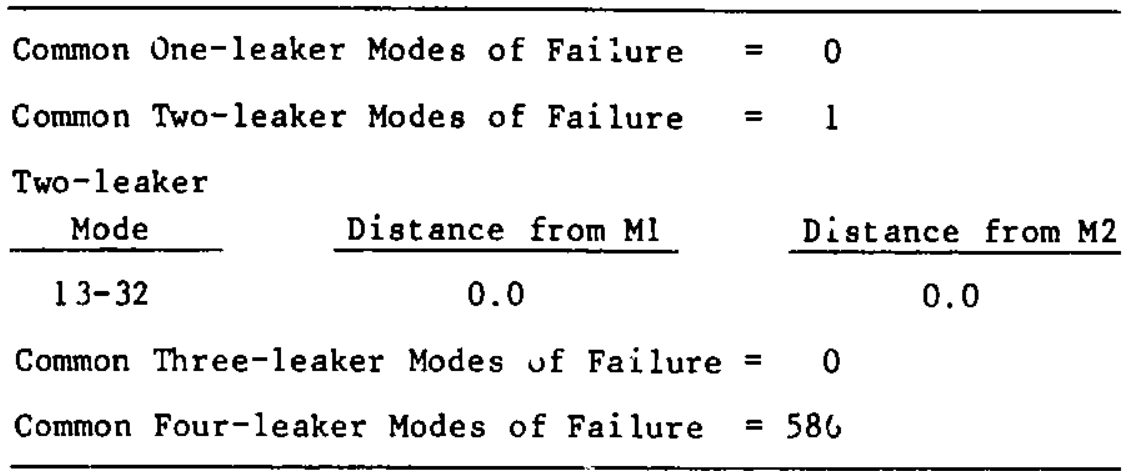

These results reveal that, if one could be reasonably confident that less than four tags were in the system, re could conclude that assemblies 13 and 32 both contain leakers and that they are the only active leakers in the core. If, on the other hand, there is even a remote possibility chat four tags might be in the system simultaneously, then it cou? be concluded from this example that unambiguous identification with only two measured samples would be impossible.

\section{Example 4}

In this example, we consider a somewhat more complicated situation involving a double simultaneous $t, g$ release in the presence of natural background gas. This example will also demonstrate how information from POLYFAIL can be used to resolve a problem of triple simultaneous failure. This is so because, as discussed in Sec. V, the background tag is treated simply as an additional suspect tag in POLYFAIL.

To simulate a double tag release in the presence of natural background gas, we assumed that tags from assemblies 20 and 40 were both released during a perind of time that the ratio of background gas to $t a g$ gas in the cover gas varied from 15 to $20 \%$. Three points were selected along the calculated mixednode trajectory to represent three possible measured tag nodes. As in Example 2, the components of each of the simulated measured sags were then shifted (this time by $4 \%$ ) to simulate the effects of experimentai-error displacement. Table XVI gives the resulting compositions of the measu:ed nodes and the ratio of natural background gas to tag gas assumed present in each.

TABLE XVI. Compositions of Simulated Measured Tag Nodes Used in Example 4

\begin{tabular}{ccccc}
\hline $\begin{array}{c}\text { Measured } \\
\text { Node }\end{array}$ & $78_{\mathrm{Kr}} / 80_{\mathrm{Kr}}$ & $82 \mathrm{Kr} / 80 \mathrm{Kr}$ & $129 \mathrm{Xe} /{ }^{126} \mathrm{Xe}$ & $\begin{array}{c}\text { Background } / \text { Tag } \\
\text { Rat i o }\end{array}$ \\
\hline M1 & 0.25425 & 3.9668 & 0.01666 & 15.5 \\
M2 & 0.27772 & 3.9606 & 0.016329 & 17.7 \\
M3 & 0.29651 & 3.9620 & 0.01600 & 19.6 \\
\hline
\end{tabular}


Table XVII summarizes the results of the three- and four-leaker rankings produced by POLYFAIL for the three measured nodes. The distance parameter shown in the second column is the value of $\mathrm{H}$ as computed by $\mathrm{Eq} .23$ for the postulated mode of failure 20-40-101. (Recall that the characteristic tag ratios for natural background gas are stored with tag ID 101.)

TABLE XVII. Summary of Computed Results

from POLYFAIL for Example 4

\begin{tabular}{cccc}
\hline $\begin{array}{c}\text { Measured } \\
\text { Node }\end{array}$ & $\begin{array}{c}\text { Distance } \\
\text { Parameter }\end{array}$ & $\begin{array}{c}\text { Number of More Likely } \\
\text { Three-leaker } \\
\text { Modes of Failure }\end{array}$ & $\begin{array}{c}\text { Number of Feasible } \\
\text { Four-leaker } \\
\text { Modes of Failure }\end{array}$ \\
\hline M1 & 6.67 & 481 & 28261 \\
M2 & 6.69 & 866 & 21556 \\
M3 & 6.70 & 619 & 25179 \\
\hline
\end{tabular}

The results given in the third column of Table XVII reveal that, for all three of the simulated measured tags, the postulated mode of failure was actually ranked quite poorly. For measured node Ml, for instance, the three-tag combination 20-40-101 was ranked 482 in the list of the most likely threeleaker modes of failure. These observations indicate that, if one were given only one measured sample, Ml, M2, or M3, a positive identification of the correct mode of failure would be highly unlikely.

Comparison of the feasible modes of failure for $M 1, M 2$, and $M 3$ reveals that there are no common one- or two-leaker. modes of failure, the most likely common three-leaker mode of failure is the combination 20-40-101, and there were over 200 common four-leaker modes of failure.

We can conclude from these results that if one could be reasonably certain that only two assembly-tag releases had occurred (which may be evident from fission-gas ${ }^{32}$ or delayed-neutron ${ }^{33}$ analyses), then three measured tag samples would be sufficient to eliminate the degenerate one-, two-, and three-leaker modes of failure and identify combination 20-40-101 as the most likely mode of failure. If, however, it were possible that four tags were in the system simultaneously, then identification of a unique mode of failure rron only three measured tag nodes would not be possible. 


\section{APPENDIX A \\ Proof of Geometric-invariance Property for Ratio-weighting Procedure}

We establish here by rigorous mathematical proof that application of the ratio-weighting scheme devised in Sec. IIL.D does not violate the inherent linearity of tag-ratio space; Specifically, we seek to establish that the values of the components of $\overrightarrow{\mathrm{C}}_{4}$ as computed by the tetrahedral simplex method remain invariant with respect to the ratio-weighting procedure. The proof requires the following definition and theorems from any standard text on matrix algebra (see, e.g., Ref. 25).

Definition: Two matrices $\|A\|$ and $\|B\|$ are conformable in the order \|A $\|$ $\|B\|$ if and only if the number of columns in $\|A\|$ is equal to the number of rows in $\|B\|$.

Theorem 1: For suitably conformable matrices, multiplication is associative, i.e.,

$$
\|A\|(\|B\|\|C\|)=(\|A\|\|B\|)\|C\|
$$

Thenrem 2: If $\|A\|$ and $\|B\|$ are nonsingular square matrices, then

$$
(\|\mathrm{A}\|\|\mathrm{B}\|)^{-1}=\|\mathrm{B}\|^{-1}\|\mathrm{~A}\|^{-1} \text {. }
$$

Theorem 3: The product of a nonsingular matrix $\|\Delta\|$ and its reciprocal in either order is a unit matrix, i.e.,

$$
\|A\|\|A\|^{-1}=\|A\|^{-1}\|A\|=\|I\| \text {, }
$$

where

$$
\|I\|=\left\|\begin{array}{llll}
1 & 0 & \ldots & 0 \\
0 & 1 & \ldots & 0 \\
\cdot & . & \ldots & . \\
0 & 0 & \ldots & 1
\end{array}\right\| \text {. }
$$

We begin with Eq. 20, which may be expressed concisely in the form

$$
\|A\|_{4}=\|B\|
$$

where $\|A\|$ is the $4 \times 4$ matrix of coefficients and $\|B\|$ is a $4 \times 1$ column matrix. The solution of Eq. A.5 for $\vec{C}_{4}$ is

$$
\vec{C}_{4}=\|\mathrm{A}\|^{-1}\|\mathrm{~B}\|
$$


It is required to show that Eq. A.6 is invariant with respect to the ratio-weighting procedure introduced in Sec. VII.D. That procedure involves premultiplying both $\|A\|$ and $\|B\|$ by a weighting matrix $\|W\|$, where

$$
\|w\|=\left(\begin{array}{cccc}
w_{1} & 0 & 0 & 0 \\
0 & w_{2} & 0 & 0 \\
0 & 0 & w_{3} & 0 \\
0 & 0 & 0 & 1
\end{array}\right) .
$$

Thus, after application of the weighting procedure, Eq. A.6 would become

$$
\vec{C}_{4}=(\|\mathrm{W}\| \quad\|\mathrm{A}\|)^{-1}(\|\mathrm{~W}\| \quad\|B\|)
$$

By application of Theorem 2 to the expression in the first set of parentheses, we have

$$
\overrightarrow{\mathrm{C}}_{4}=\|\mathrm{A}\|^{-1}\|\mathrm{w}\|^{-1}(\|\mathrm{w}\|\|\mathrm{B}\|) \text {. }
$$

Since matrix multiplication is associative (Theorem 1), we may rewrite Eq. A.9 in the form

$$
\mathrm{C}_{4}=\|\mathrm{A}\|^{-1}\left(\|\mathrm{~W}\|^{-1}\|\mathrm{~W}\|\right)\|B\| .
$$

Applying Theorem 3 to the product in parentheses, we obtain

$$
\overrightarrow{\mathrm{C}}_{4}=\|\mathrm{A}\|^{-1}\|\mathrm{I}\|\|\mathrm{B}\| \text {. }
$$

Hence, by definition of the unit matrix (see Eq. A.4), Eq. A.1l reduces to

$$
\vec{C}_{4}=\|\mathrm{A}\|^{-1}\|\mathrm{~B}\| \text {, }
$$

which is equivalent to Eq. A.6, as asserted. 


\section{APPENDIX B}

Isotopic Ratios for Reference System of Tag Nodes

Table B.1 lists the values of the isotopic ratios for the reference system of tag nodes used to demonstrate operation of POLYFAIL in Sec. VIII. The thrt. primary ratios $\mathrm{Rl}, \mathrm{R} 2$, and $\mathrm{R} 3$ represent the respective ratios of $78 \mathrm{Kr}$ to $80 \mathrm{Kr},{ }^{82} \mathrm{Kr}$ to $80 \mathrm{Kr}$, and $129 \mathrm{Xe}$ to $126 \mathrm{Xe}$. The values shown were obtained from Ref. 22 .

Figures B.1-B.3 give three-dimensional plots of the reference system of tag nodes.

TABLB B.1. Tag Ratios for Syotem of Tag Nodes Used in Example POLYFAIL Calculationg

\begin{tabular}{|c|c|c|c|c|c|c|c|}
\hline T 88 ID & $\mathrm{RI}$ & 22 & R3 & Tag ID & R1 & 82 & $\mathbf{R 3}$ \\
\hline 1 & 0.1798 & 3.733 & 0.01000 & 52 & 0.2523 & 2.297 & 0.01822 \\
\hline 2 & 0.1826 & 3.657 & 0.01220 & 53 & 0.4659 & 3.152 & 0.00985 \\
\hline 3 & 0.1815 & 3.719 & 0.01497 & 54 & 0.4738 & 3.089 & 0.01229 \\
\hline 4 & 0.1822 & 3.640 & 0.01810 & 55 & 0.4704 & 3.128 & 0.01485 \\
\hline 5 & 0.2279 & 4.351 & 0.00975 & 56 & 0.4808 & 3.057 & 0.01833 \\
\hline 6 & 0.2277 & 4.607 & 0.01250 & 57 & 0.5807 & 3.313 & 0.00986 \\
\hline 7 & 0.2262 & 4.636 & 0.01520 & 58 & 0.5857 & 3.304 & 0.01236 \\
\hline 8 & 0.2242 & 4.612 & 0.01860 & 59 & 0.5788 & 3.333 & 0.01467 \\
\hline 9 & 0.1788 & 4.671 & 0.01015 & 60 & 0.5909 & 3.290 & 0.01835 \\
\hline 10 & 0.1813 & 4.555 & 0.01253 & $6 !$ & 0.3894 & 2.680 & 0.01020 \\
\hline 11 & 0.1781 & 4.636 & 0.01479 & 62 & 0.3958 & 2.628 & 0.01228 \\
\hline 12 & 0.1816 & 4.538 & 0.01874 & 63 & 0.3895 & 2.671 & 0.01502 \\
\hline 13 & 0.2935 & 4.527 & 0.01003 & 64 & 0.3997 & 2.614 & 0.01832 \\
\hline 14 & 0.2938 & 4.446 & 0.01221 & 65 & 0.3117 & 2.235 & 0.01008 \\
\hline 15 & 0.2923 & 4.525 & 0.01487 & 66 & 0.3176 & 2.200 & 0.01228 \\
\hline 16 & 0.2946 & 4.427 & 0.01848 & 67 & 0.3152 & 2.239 & 0.01465 \\
\hline 17 & 0.2228 & 3.567 & 0.00999 & 68 & 0.3224 & 2.190 & 0.01842 \\
\hline 18 & 0.2248 & 3.564 & 0.01218 & 69 & 0.4906 & 2.519 & 0.01004 \\
\hline 19 & 0.2218 & 3.640 & 0.01502 & 70 & 0.4888 & 2.511 & 0.01218 \\
\hline 20 & 0.2248 & 3.533 & 0.01861 & 71 & 0.5008 & 2.493 & 0.01498 \\
\hline 21 & 0.3696 & 4.262 & 0.00991 & 72 & 0.5018 & 2.471 & $0 .:: 823$ \\
\hline 22 & 0.3691 & 4.223 & 0.01218 & 73 & 0.6130 & 2.675 & 0.00998 \\
\hline 23 & 0.3661 & 4.255 & 0.01464 & 74 & 0.6054 & 2.674 & 0.01225 \\
\hline 24 & 0.3687 & 4.191 & 0.01844 & 75 & 0.6186 & 2.649 & 0.01500 \\
\hline 25 & 0.2960 & 3.634 & 0.00998 & 76 & 0.6291 & 2.615 & 0.01819 \\
\hline 26 & 0.3045 & 3.523 & 0.01216 & 77 & 0.3930 & 2.125 & 0.01021 \\
\hline 27 & 0.2973 & 3.639 & 0.01455 & 78 & 0.3924 & 2.115 & 0.01222 \\
\hline 28 & 0.2995 & 3.558 & 0.01860 & 79 & 0.3950 & 2.142 & 0.01490 \\
\hline 29 & 0.2475 & 2.952 & 0.00996 & 80 & 0.4053 & 2.052 & 0.01825 \\
\hline 30 & 0.2511 & 2.858 & 0.01225 & 81 & 0.2239 & 3.600 & 0.02209 \\
\hline 31 & 0.2493 & 2.928 & 0.01463 & 82 & 0.2971 & 3.648 & 0.02219 \\
\hline 32 & 0.2584 & 2.809 & 0.01862 & 83 & 0.2492 & 2.929 & 0.02228 \\
\hline 33 & 0.4563 & 3.908 & 0.00985 & 84 & 0.3697 & 3.390 & 0.02229 \\
\hline 34 & 0.4614 & 3.861 & 0.01229 & 85 & 0.3120 & 2.807 & 0.02198 \\
\hline 35 & 0.4592 & 3.885 & 0.01489 & 86 & 0.2006 & 2.880 & 0.02214 \\
\hline 36 & 0.4572 & 3.814 & 0.01862 & 87 & 0.6855 & 2.137 & 0.01237 \\
\hline 37 & 0.3682 & 3.389 & 0.00983 & 88 & 0.2694 & 1.792 & 0.01221 \\
\hline 38 & 0.3728 & 3.348 & $0.0: 209$ & 89 & 0.7232 & 1.744 & 0.01240 \\
\hline 39 & 0.3698 & 3.382 & 0.01461 & 90 & 0.5108 & 1.436 & 0.01224 \\
\hline 40 & 0.3730 & 3.293 & 0.01853 & 91 & 0.3499 & 1.687 & 0.01208 \\
\hline 41 & 0.2006 & 2.867 & 0.00989 & 92 & 0.4660 & 1.785 & 0.01239 \\
\hline 42 & 0.2038 & 2.794 & 0.01219 & 93 & 0.4866 & 1.986 & 0.01514 \\
\hline 43 & 0.2012 & 2.860 & 0.01485 & 94 & 0.2647 & 1.841 & 0.01493 \\
\hline 44 & 0.2030 & 2.827 & 0.01860 & 99 & 0.4876 & 1.984 & 0.01023 \\
\hline 45 & 0.3133 & 2.807 & 0.01006 & 96 & 0.2647 & 1. 2 & 0.01005 \\
\hline 46 & 0.3149 & 2.747 & 0.01214 & 97 & 0.6047 & 2.088 & 0.01020 \\
\hline 47 & 0.3103 & 2.777 & 0.01461 & 98 & 0.7464 & 2.175 & 0.01007 \\
\hline 48 & 0.3151 & 2.735 & 0.01825 & 99 & 0.3397 & 1.739 & 0.01005 \\
\hline 49 & 0.2480 & 2.360 & 0.00981 & 100 & 0.7283 & 1.786 & 0.01006 \\
\hline 30 & $\begin{array}{l}0.2530 \\
0.2479\end{array}$ & $\begin{array}{l}2.313 \\
2.359\end{array}$ & $\begin{array}{l}0.01226 \\
0.01467\end{array}$ & 101 & 0.1565 & 5.160 & 0.00346 \\
\hline
\end{tabular}




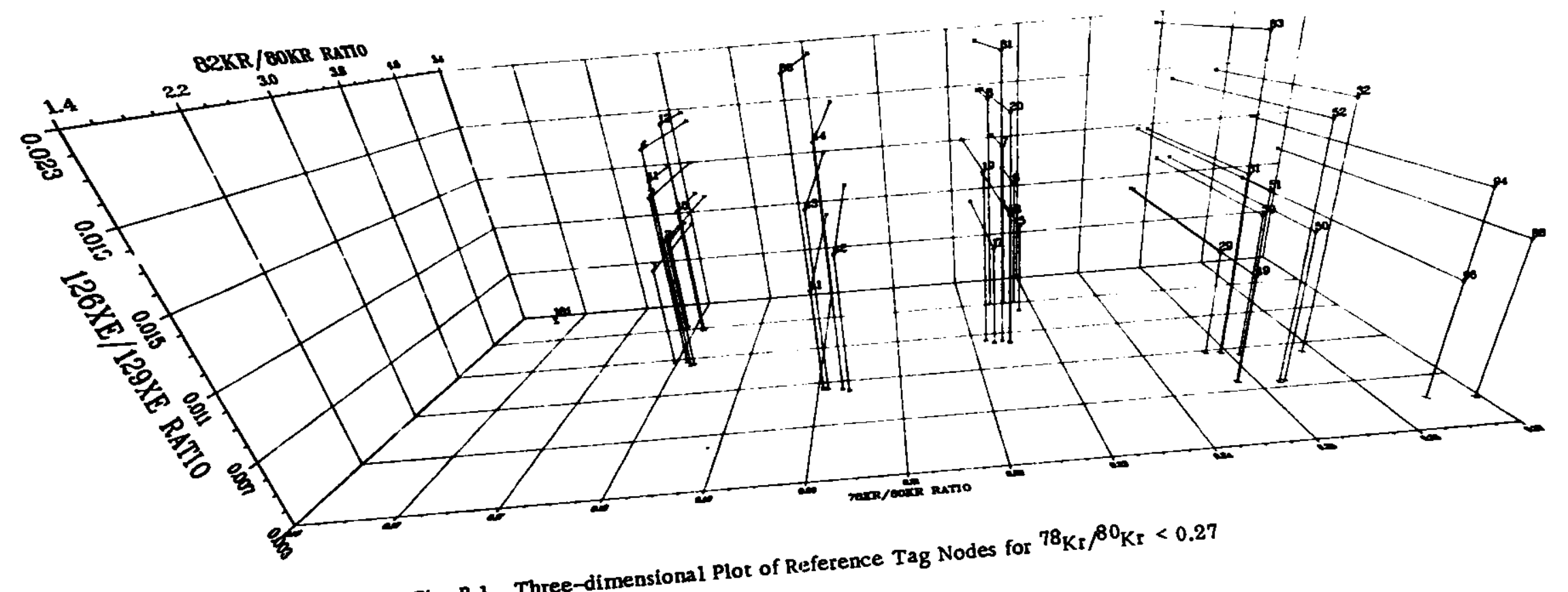




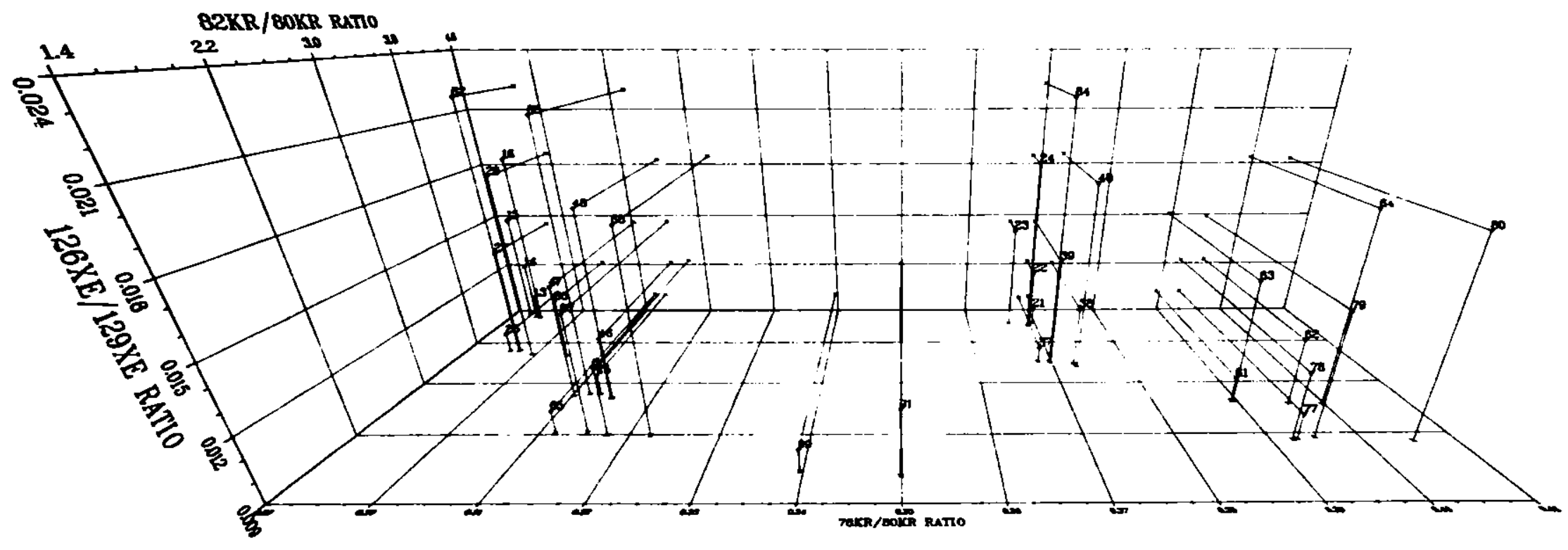

Fig. B.2. Three-dimensional Plot of Reference Tag Nodes for $0.27<78_{\mathrm{Kr}} / 80_{\mathrm{Kr}}<0.41$ 


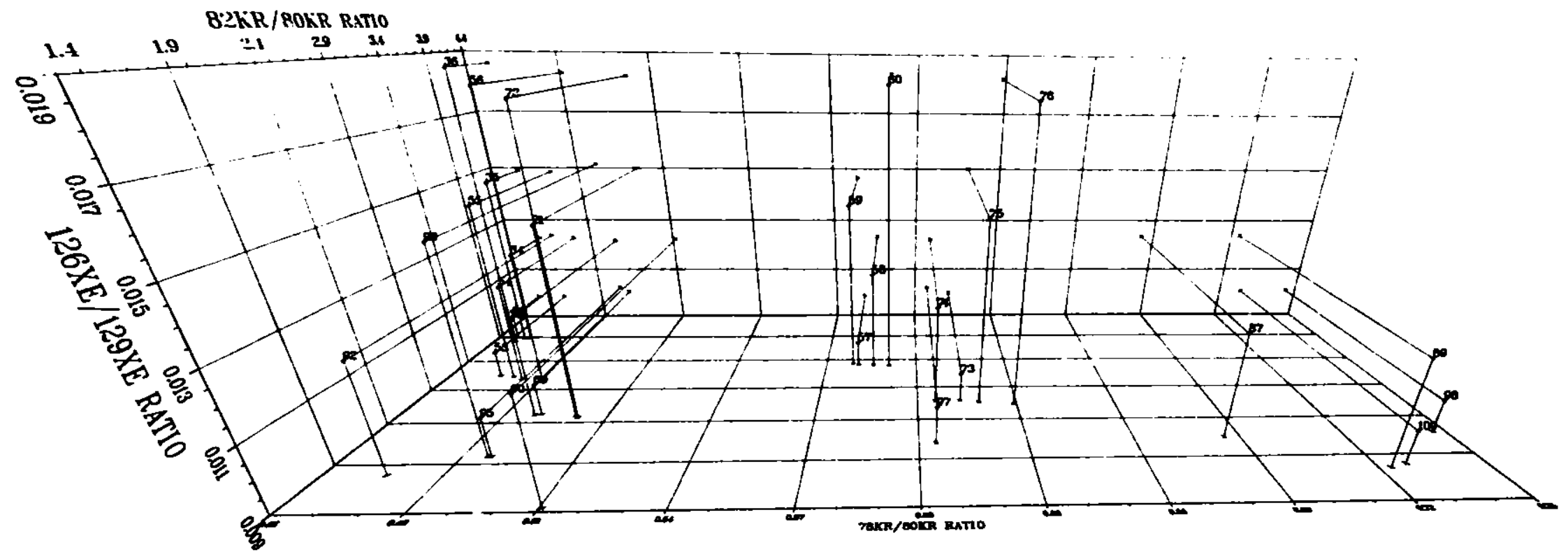

Fig. B.3. Three-dimensional Plot of Reference Tag Nodes for ${ }^{78} \mathrm{Kr} / 80_{\mathrm{Kr}}>0.41$ 


\section{REFERENCES}

1. Reactor Develonment Progress Report, September 1970, ANJ-7742, Argonne National Laboratory, p. 82 (nct 23, 1970).

2. N. J. McCormick, Gas Tag Identification of Failed Fuel-II. Resolution Retween sinale and Multiple Failures, Nucl. Technol. 24, 156 (1974).

3. J. D. B. Lambert, B. Y. C. So, F. S. Kirn, J. R. Armstrong, E. R. Ebersole, and M. T. Laug, Recent Improvements in Identifying Fission Product Sources in the Fxperimental Rreeder Reactor II, Nuc1. Technol. 39, 275 (1978).

4. K. C. Gross, Gas Tagging for Failed Fuel Identification in LWR's, Ph.D. thesis, Univ. of Cincinnati (1977).

5. K. C. Gross and C. Passerello, Rampentric-coordinates Technique for Itentification of Simultaneous Fuel. Failure with Gas Tagaing, Nucl. Sci. Eng. 75, I (July 1980).

6. R. B. Kidman, Tag Gas Bumup Based on 3-dimensional Analysis, HEDL-TME 7458, Hanford Engineering Development Laboratory (1974).

7. R. E. Schenter and F. Schmittroth, Noble Gas Cross Section Calculations for Fuel Failure Location Studies, Trans. Am. Nucl. Soc. 15, 462 (June 1972).

8. R. B. Kidman, FTR Tag Bumup, Trans. Am. Nuc1. Soc. 20, 452 (June 1975).

9. K. C. Gross, Concentric-sphere Design for Spacing of Tag-gas Isotopic Ratios, Nuc1. Technol. 45, 195 (Sept 1979).

10. N. J. McCormick and R. E. Schenter, Gas Tag Identification of Failed Fuel-I. Synergistic Use of Inert Gases, Nucl. Technol. 24, 149 (1974).

11. K. C. Gross, W. Roos, and A. Shapiro, Dynamic Behavior of Tag Gas Isotopes in PWR Primary Coolant after Fuel Failure, Trans. Am. Nuc1. Soc. 24, 396 (1976).

12. K. C. Gross, A. Shapiro, and L. E. Eckart, Gas Tagging in a PWR, Trans. Am. Nucl. Soc. 15, 429 (1972).

13. I. Fried, Some Aspects of the Natural Coordinate System in the Finite Filement Method, AIAA J. ?, 1366 (July 1969).

14. E. M. Patterson, Topoloay, Oliver and Boyd, Edinburgh (1959).

15. F. G. Lannoy, Trianqular Finite E7ements and Numerisal Integration, Comput. Struct. 7, 613 (1977).

16. F. Schmittroth, A Probabilistic Method for Identifying Failed Fuel Elements Using A Gas Tag system, Nucl. Sci. Eng. 52, 95 (1973).

17. J. F. DeClue, N. J. McCormick, and F. Schmittroth, Probabilistic Identification of Failed Core Assemblies from Gas Tag Releases, Trans. Am. Nucl. Soc. 27, 686 (Nov 1977). 
18. B. Y. C. So, Identifying a Second Xenon Tag in ERR-II Cover Gas in the Presence of a First Tag, Trans. Am. Nucl. Soc. 24, 424 (Nov 1976).

19. J. E. Marsden and A. J. Tromba, Vector Calculus, W. H. Freeman and Co., San Francisco (1970).

20. L. Mirsky, Linear AZgehra, Oxford Book Co., Inc., New York (1955).

21. J. A. Figg, R. E. Schenter, and J. F. DeClue, Gas Tag Designs for a Generic LMFRR, Trans. Am. Nucl. Soc. 27, 580 (1977).

22. J. A. Figg, N. J. McCormick, R. E. Schenter, F. Schmittroth, J. F. DeClue, and F. E. Holt, Gas Tag Location Systems for FFTF and CRRRP Failed Assemblies, HEDL-TME-14, Hanford Engineering Development Laboratory ( July 1978).

23. K. H. Huebner, The Finite Flement Method for Fnaineers, Wiley \& Sons, New York (1975).

24. M. R. Speigel, Advanced Mathematics for Fingineers and Scientists, Schaum's outline Series, Ch. 15, McGraw-Hil1, New York (1971).

25. C. R. Wylie, Jr., Advanced Engineerina Mathematics, Third Edition, McGraw-Hi11, New York (1966).

26. G. Arfken, Mathematical. Methods for Physicists, Second Edition, Chapter 1, Academic Press, New York (1970).

27. Y. Bard, Nonlinear Parameter Fstimation, Chapter IV, Academic Press, New York (1974).

28. P. B. Liebelt, An Introduction to Optimal Estimation, Chapter 5, Addi son-Wesley, Reading, Mass. (1967).

29. K. C. Gross and B. Y. C. So, Incorporation of I6otopic Weightinq Factors for Improved Gas-tagging Analutical Procedures, Trans. Am. Nuc1. Soc. 28, 534 (1978).

30. K. Bathe and E. L. Wilson, Numerical Methods in Finite Filement Analysis, Prentice-Hall, Englewood Clilifs, N.J. (1976).

31. A. d'Abro, The Evolution of Scientific Thought from Newton to Einstein, Second Edition, Dover (1950).

32. B. Y. C. So, K. C. Gross, J. D. B. Lambert, F. S. Kirn, E. R. Ebersole, and M. T. Laug, Fission-product Releases to the Primary System of FBR-II from April 1977 to May 1978, ANL-79-27 (July 1979).

33. K. C. Gross and R. V. Strain, Delayed Neutron Signal Characterization in a Fast Reactor, Nuc1. Sci. Eng. 76(2), 163-174 (Nov 1980). 